\title{
The influence of atherosclerotic plaques on the pharmakokinetics of a drug eluted from bioabsorbable stents
}

\author{
Jahed Naghipoor ${ }^{1, *}$, José A. Ferreira ${ }^{2}$, Lino Gonçalves ${ }^{3}$, Paula de Oliveira ${ }^{2}$ and \\ Timon Rabczuk ${ }^{1}$ \\ ${ }^{1}$ Institute of Structural Mechanics, Bauhaus University of Weimar, 99423, Weimar, \\ Germany \\ ${ }^{2}$ CMUC, Department of Mathematics, University of Coimbra, 3001-454, Coimbra, \\ Portugal \\ ${ }^{3}$ Department of Cardiology CHUC-HG, Hospital Center of University of Coimbra, \\ Coimbra, Portugal
}

\begin{abstract}
In this paper the effect of plaque composition, on the accumulation of drug released by a drug eluting stent, is analyzed. The mathematical model is represented by two coupled systems of partial differential equations that describe the pharmacokinetics of drug in the stent and in the arterial wall. The influence of the stiffness and porosity of soft and hard plaques is studied. A case study based on optical coherence tomography images is also included.
\end{abstract}

Keywords: Atherosclerosis plaque, porosity, drug eluting stent, numerical simulation

\section{Introduction}

Cardiovascular diseases are the leading cause of mortality in the world. They are responsible for the death of 17.3 million people and this number is expected to increase to more than 23.6 million by 2030. Among cardiovascular diseases, atherosclerosis that is characterized by the narrowing and hardening of some arteries that start thickening and eventually occlude, is the most common. This process normally happens over a period of 50 to 60 years and seems to get particularly severe with age $(23)$.

Atherosclerosis is due to cholesterol deposition, caused by inflammation of the intima which is the innermost layer of the arterial wall. The main causes for the disease are mainly high blood

${ }^{*}$ Corresponding author. Tel.: +49 3643584512 .

E-mail: jahed.naghipoor@uni-weimar.de 
pressure, diabetes, high levels of cholesterol, smoking, age and genetic background. The evolution of atherosclerosis plaque can be generally divided into four stages: endothelial injury, oxidation of low-density lipoprotein (LDL), inflammatory process and calcification. Once a plaque is formed, it is covered by a fibrous cap, consisting of elastin, collagen and smooth muscle cells. Rupture of this cap can lead to serious events such as myocardial infarction and sudden death. To prevent this as well as the serious occlusion of the blood lumen, different treatments have been developed. These treatments have moved from invasive techniques such as coronary artery bypass grafting to more safe and noninvasive techniques like percutaneous coronary intervention. Balloon angioplasty was the first noninvasive and nonsurgical technique of mechanically widening the narrowed or obstructed arteries. After many years of clinical experience, balloon angioplasty is still far from being the perfect technique to control the occlusion of the lumen effectively. A common problem called restenosis, that is the re-narrowing of the blood lumen after intervention, is the main drawback of balloon angioplasty. Restenosis can occur when the lumen of the vessel becomes narrower with the proliferation of smooth muscle cells a few weeks after coronary angioplasty procedure. To overcome this drawback complementary techniques like implantation of metallic or polymeric stents are now of common use. These are several types of stents. The first generation of stents Bare Metal Stents, BMS, are tiny expandable mesh tubes made by stainless steel or other metallic alloys. In spite of obvious advantages BMS were associated to rates of restenosis of $20 \%$ to $30 \%$ ([12]).

Although BMSs have defined the standard of care for atherosclerotic plaques obstruction, stents coated by polymers, the so-called Drug Eluting Stents, DESs, promise significant patient benefits. A DES is actually a BMS coated by a polymer containing an anti-proliferative agent which is released gradually over the course of weeks to months after insertion of the stent. It provides sustained inhibition of the neointimal proliferation as a response to endothelial injury. DES has three principal components, namely the stent platform (strut), the polymer coating and the drug. The drug is dispersed in the polymer's coating and diffuses into the arterial wall. The first DESs were designed with nondegradable polymer coatings. As a large number of clinical studies ([1,28]) concluded that they perpetuate local vascular inflammation, biodegradable polymers that vanish from the vascular surface after a period of time, are now used in the coatings ([29]).

Different polymers have attracted considerable interest as base materials for biomedical applications such as biodegradable stents due to its biocompatibility, tailored biodegradation rate, approval for clinical use in humans and potential to modify surface properties to provide better interaction with biological materials $([13)$.

In this paper, we study DES coated with polylactic acid (PLA) where a drug is dispersed. The drug at first is in the solid phase and then dissolves in the presence of the plasma. Changes, in the porosity and the biodegradation rate of the polymer as the process evolves, are considered in the mathematical models.

In previous papers ([10,18] ) by some of the authors, the influence of the arterial stiffness, on the drug release, was studied. In this paper, while keeping in the model the stiffness of the arterial wall, a more realistic description is considered, that includes location, geometry and composition of different types of atherosclerotic plaques. We consider an arterial wall containing both lipid and hard plaques with a non-diffusible calcified core. The viscoelastic properties of the arterial wall are also considered as an indicator of the heterogeneous stiffness of the vessel walls. The reversible nature of bindings between the drug and specific fixed sites inside the arterial wall is 
also taken into account. The behaviors of two different molecules, Sirolimus and Paclitaxel, which are nowadays the most used in first generation commercialized drug eluting stents, are compared. These last years many clinical researchers have carried on studies to compare the clinical results of the two stents that are based on these drugs. The battle is still ongoing. We are aware that a mathematical model can not describe the huge complexity of their in vivo pharmackokinetics. However we believe that our results can give a contribution to better understand the absorption of drug concentration in the vessel walls.

The aim of the paper is to show that the sorption of drug by the vessel wall is a spatially heterogeneous process, highly dependent on the local composition of the plaques. As the drug eluted from the stent is an antiproliferative one, we believe that the model presented here can act like a predictive tool of the location of restenosis areas.

The paper is organized as follows. Section 2 is devoted to the mathematical modeling of the problem. Numerical simulations are included in Section 3. In Section 4, some conclusions are presented.

\section{Mathematical modeling}

\subsection{General description}

Let us consider the two dimensional domain in Figure 1 representing a cross section of a blood vessel where a drug eluting stent has been inserted. The vessel walls are heterogeneous containing calcified and lipid plaques. The two dimensional domain $S \subset \mathbb{R}^{2}$ represents a drug eluting stent with an impermeable metallic core; $V \subset \mathbb{R}^{2}$ represents the healthy part of the arterial wall, while $S P$ and $H P$ represent soft and hard plaques, respectively. It is expected that newly implanted stents will be located at the lumen boundary.

In Figure 1 we consider two possible struts placements: in the lumen boundary, partially immersed in the healthy part of the vessel wall and completely immersed in a soft lipid plaque. The contribution, to drug concentration in the vessel wall, of each type of strut location is simulated. Malapposition of struts can also occur leading to a huge drug loss of drug that is completely released in the blood flow. In Section 3, a real situation, obtained from an optical coherence tomography (OCT) image, where some struts are malapposed will also be considered. The differences in the concentration of drug eluted from the stents in different locations show that placement evaluation is important in a clinical follow-up analysis.

The hard plaque is composed of fatty laden foam cells and a calcium core. We assume that the calcium core is so stiff that it is impermeable to any diffusible molecules. The domain of such calcified core is excluded from the computational domain. The internal boundary of the vessel is

represented by $\Gamma_{\text {wall-lumen }}$ while the external boundary, $\Gamma_{a d v}$, represents the interface between the intima and the adventitia. 


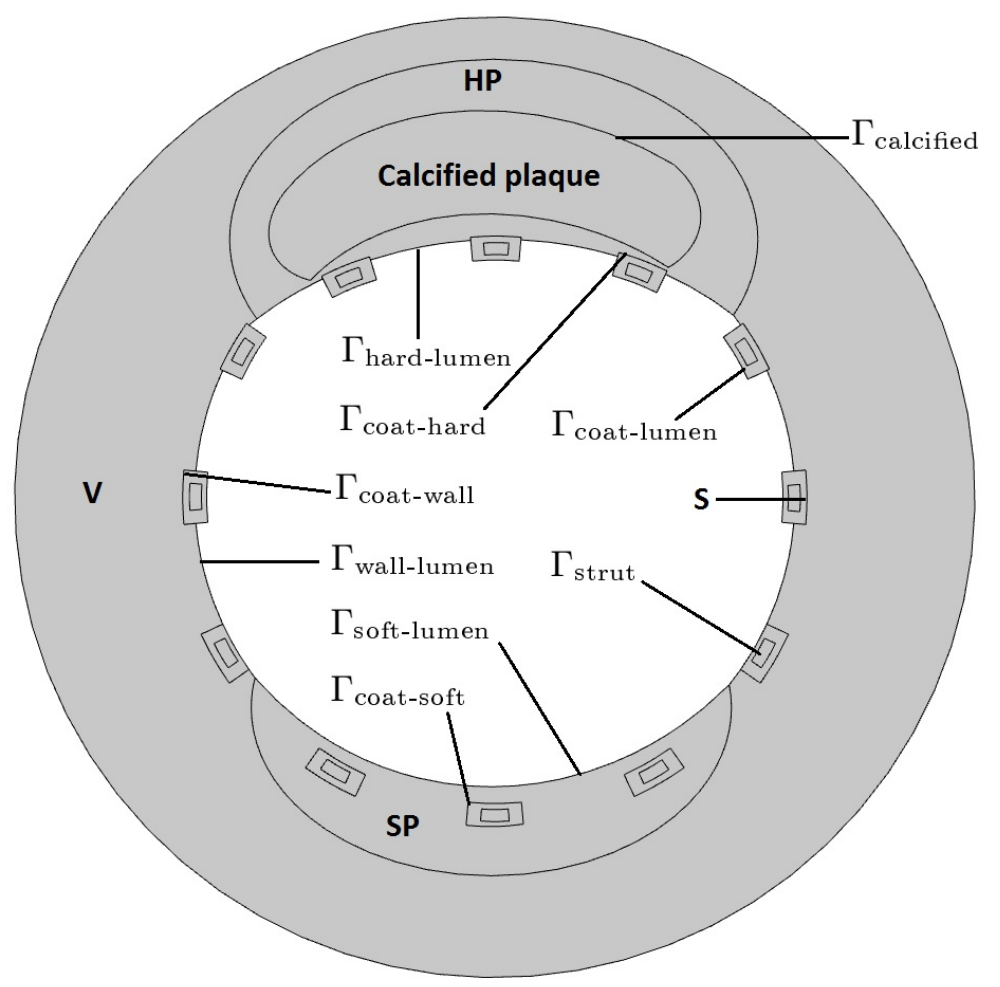

Figure 1: Cross section of a vessel where a stent is inserted; stent (S), vessel wall (V), soft plaque (SP) and hard plaque (HP).

This work presents a mathematical model that describes the integrated process of drug release from coating and subsequent distribution and drug pharmacokinetics in the arterial wall. A mechanistic model for drug kinetics in the coating is adopted: it couples the drug diffusion with the degradation and erosion, complemented with an arterial wall model where binding is included in the pharmacokinetics of the drug. A mass transport process and a series of chemical reactions are responsible for the degradation of the polymer and the release of the drug.

To describe the influence of the heterogeneity of the vessel wall the differences in stiffness and porosity of the plaques are taken into account.

We introduce the following notations:

$$
\begin{aligned}
& \mathcal{M}_{S}=\{W, P, O, L, S D, D D\}, \mathcal{M}_{j}=\{W, O, L, B D, U D\}, j=V, S P, H P, \\
& \mathcal{C}_{j}=\left(C_{m, j}\right)_{m \in \mathcal{M}_{j}}, j=S, V, S P, H P
\end{aligned}
$$

where $W, P, O, L, S D$ and $D D$ stand, respectively, for plasma, PLA, oligomers, lactic acid, solid and dissolved drug in the stent, while $B D$ and $U D$ stand respectively for bound and unbound drugs in the healthy wall and the plaques. In Table 1 we summarize the previous notations. 


\begin{tabular}{|c|c|c|c|c|c|c|c|c|}
\hline & Plasma & Polymer & Oligomers & $\begin{array}{c}\text { Lactic } \\
\text { Acid }\end{array}$ & $\begin{array}{c}\text { Solid } \\
\text { drug }\end{array}$ & $\begin{array}{c}\text { Dissolved } \\
\text { drug }\end{array}$ & $\begin{array}{c}\text { Bound } \\
\text { drug }\end{array}$ & $\begin{array}{c}\text { Unbound } \\
\text { drug }\end{array}$ \\
\hline S & W & P & O & L & SD & DD & - & - \\
\hline V & W & - & O & L & - & DD & BD & UD \\
\hline SP & W & - & O & L & - & DD & BD & UD \\
\hline HP & W & - & O & L & - & DD & BD & UD \\
\hline
\end{tabular}

Table 1: Concentration variables in the stent (S), vessel wall (V), soft and hard plaques (SP and HP respectively).

\subsection{Drug release in the stent}

Reaction-diffusion in the polymer Drug release from a resorbable polymer coating depends on both polymer degradation and drug molecular diffusional transport. Two main reactions are responsible for the degradation of PLA into smaller molecules. The first reaction is the hydrolysis of the PLA producing oligomers which have smaller molecular weights $M_{W}, 2 \times 10^{4} \mathrm{~g} / \mathrm{mol} \leq M_{W} \leq$ $1.2 \times 10^{5} \mathrm{~g} / \mathrm{mol}$. It is assumed that all of these oligomers have similar diffusion coefficients when they diffuse through the coated stent $([21])$. The second reaction is the hydrolysis of the oligomers producing lactic acid with molecular weight $M_{W} \leq 2 \times 10^{4} \mathrm{~g} / \mathrm{mol}$. The lactic acid generated by this reaction is assumed to have a catalytic effect on further degradation of the PLA ([21]). These reactions are schematically represented by

$$
\begin{aligned}
& C_{W, S}+C_{P, S} \stackrel{k_{P W, S}}{\longrightarrow} C_{O, S}+C_{L, S}, \\
& C_{W, S}+C_{O, S} \stackrel{k_{O W, S}}{\longrightarrow} C_{L, S},
\end{aligned}
$$

where $C_{W, S}, C_{P, S}, C_{O, S}$ and $C_{L, S}$ denote the concentrations of plasma, PLA, oligomer and lactic acid in the polymeric coating of the stent respectively.

The diffusion coefficients of the different species, oligomers, lactic acid and dissolved drug in the polymer vary during the degradation process. As the polymer degradation proceeds, diffusional paths are opened through the polymer matrix pores, allowing dissolved drug molecules to leave the device via a degradation-controlled release $([22])$. Hence, the diffusivity coefficients in the coated stent are defined by $([8,21])$

$$
D_{m, S}=D_{m, S}^{0} \mathrm{e}^{\theta_{m, S} \frac{C_{P, S}^{0}-C_{P, S}}{C_{P, S}^{0}}} \quad \text { in } \bar{S} \times \mathbb{R}^{+}, m \in \mathcal{M}_{S}, m \neq P, S D,
$$

where $D_{m, S}^{0}, m \in \mathcal{M}_{S}, m \neq P, S D$, is the diffusivity of the respective species in unhydrolyzed polymer, $C_{P, S}^{0}$ is the concentration of polymer at $t=0$ and $\theta_{m, S}, m \in \mathcal{M}_{S}$, are experimental constants.

When the plasma diffuses through the polymeric matrix, the polymer swells and the solid drug particles are then activated. As polymer degradation proceeds new diffusional paths are opened through the polymer matrix pores. The solid drug in contact with the plasma starts to dissolve according to its thermodynamics and the kinetics of the process. The dissolved drug diffuses through the polymer matrix. The process is schematically represented by the following relation

$$
C_{S D, S}+C_{W, S} \stackrel{\kappa_{D D, S}}{\longrightarrow} C_{D D, S}
$$


where $C_{S D, S}$ and $C_{D D, S}$ stand for the concentrations of the solid drug and the dissolved drug respectively and $\kappa_{D D, S}$ is the dissolution coefficient.

Mathematical model The mass conservation equations for the plasma, the oligomer, the lactic acid and the drug assume the following form

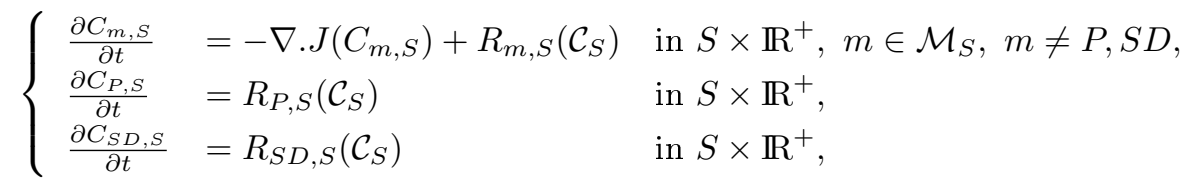

with

$$
R_{m, S}\left(\mathcal{C}_{S}\right)= \begin{cases}-\sum_{i=1,2} \mathcal{R}_{i, S}\left(\mathcal{C}_{S}\right), & \mathrm{m}=\mathrm{W} \\ -\mathcal{R}_{1, S}\left(\mathcal{C}_{S}\right), & \mathrm{m}=\mathrm{P} \\ \sum_{i=1,2}(-1)^{i-1} \mathcal{R}_{i, S}\left(\mathcal{C}_{S}\right), & \mathrm{m}=\mathrm{O} \\ \sum_{i=1,2} \mathcal{R}_{i, S}\left(\mathcal{C}_{S}\right), & \mathrm{m}=\mathrm{L} \\ \mathcal{R}_{3, S}\left(\mathcal{C}_{S}\right), & \mathrm{m}=\mathrm{DD} \\ -\mathcal{R}_{3, S}\left(\mathcal{C}_{S}\right), & \mathrm{m}=\mathrm{SD}\end{cases}
$$

and

$$
\left\{\begin{array}{l}
\mathcal{R}_{1, S}\left(\mathcal{C}_{S}\right)=k_{P W, S} C_{W, S} C_{P, S}\left(1+\alpha C_{L, S}\right) \\
\mathcal{R}_{2, S}\left(\mathcal{C}_{S}\right)=k_{O W, S} C_{W, S} C_{O, S}\left(1+\beta C_{L, S}\right) \\
\mathcal{R}_{3, S}\left(\mathcal{C}_{S}\right)=k_{D D, S} C_{W, S} C_{S D, S}\left(C_{S o l}-C_{D D, S}\right)
\end{array}\right.
$$

where $C_{S o l}$ is the solubility of the drug ([2] 16$\left.]\right)$.

In (5),

$$
J\left(C_{m, S}\right)=-D_{m, e f f} \nabla C_{m, S}, \quad m \in \mathcal{M}_{S},
$$

represents the fluxes in the polymer where $D_{m, e f f}$ is defined as in [29] by

$$
D_{m, e f f}=\frac{\left(1-\phi_{S}\right) D_{m, S}+k_{S} \phi_{S} D_{m, V}}{1-\phi_{S}+k_{S} \phi_{S}}, \quad m \in \mathcal{M}_{S} .
$$

In (9), the porosity of the polymer, $\phi_{S}$, is given by

$$
\phi_{S}=\phi_{S, 0}+\left(1-\phi_{S, 0}\right)\left(1+e^{-2 k_{P W, S t}}-2 e^{-k_{P W, S} t}\right),
$$

assuming the same density for PLA chains of different lengths $([29])$. In 10$], \phi_{S, 0}$ is the initial porosity in the polymeric coating and the expression $1+e^{-2 k_{P W, S} t}-2 e^{-k_{P W, S t}}, t \geq 0$, represents the effect biodegradation. It describes the increase of porosity as a function of time, where $k_{P W, S}$ stands for a degradation rate constant.

Initial and boundary conditions Equations (5)- 10 are completed with initial and boundary conditions. At the initial time $(t=0)$ the drug is assumed to be in the solid state and entirely contained in the polymeric coating, with a uniform distribution. The initial conditions in the coating are as follows:

$$
C_{m, S}(0)=0, \quad m \in \mathcal{M}_{S}, m \neq P, S D, C_{m, S}(0)=C_{m, S}^{0}, m=P, S D .
$$


As the metallic stent strut is impermeable to the drug and the polymer degradation products that diffuse in the coating stent, a no-flux condition is considered for the boundary surface $\Gamma_{\text {strut. Diffusable particles, }}$ oligomers, lactic acid and dissolved drug, in struts are washed out by the blood flow and are transported fast away from the region of interest. The Dirichlet boundary conditions $C_{m, S}=0, m \in \mathcal{M}_{S}, m \neq W, P$, are considered in the boundaries in contact with the blood flow. The Dirichlet boundary condition $C_{W, S}=$ $C_{W \text {,out }}$ is considered for the plasma on $\Gamma_{\text {coat-lumen }}$ where $C_{W \text {,out }}$ stands for the plasma concentration in the lumen. For struts which are immersed in the soft plaque, interface boundary conditions are prescribed. Oligomers, lactic acid and dissolved drug pass through $\Gamma_{\text {coat-wall }}, \Gamma_{\text {coat-soft }}$ and $\Gamma_{\text {coat-hard }}$ diffusing into the healthy and soft and hard plaque regions. The interface boundary conditions considered for $\Gamma_{\text {coat-wall, }}$ $\Gamma_{\text {coat-soft }}$ and $\Gamma_{\text {coat-hard }}$ will be introduced in section 2.4

\subsection{Reaction-diffusion-convection equations in the arterial wall}

We consider the notation

$$
\mathcal{M}_{j}=\{W, O, L, B D, U D\}, j=V, S P, H P,
$$

where $B D$ and $U D$ stand for the bound and unbound drugs in the arterial wall.

Chemical reactions We assume the reversible nature of the bindings between the drug and specific sites inside the arterial wall. These reactions depend on the type of drug. Bindings occur when drug and binding site hit each other, due to diffusion forces, and when the collision has the correct orientation and enough energy $([17])$. When binding has occurred, drug and binding site remain bound together for an amount of time depending on the affinity of the binding site and the drug. Binding does neither alter the properties of drug nor the properties of binding sites so the reaction is schematically represented by

$$
\text { Drug }+ \text { Binding sites } \underset{\text { dissociation }}{\stackrel{\text { association }}{\rightleftarrows}} \text { Drug-binding complex. }
$$

To define the mathematical kinetic model associated with $\sqrt{12}$, we assume that all the binding sites are equally accessible to drug. We also assume that there are not states of partial binding meaning that the binding sites are either free or attached to drug.

The drug assumes two different states: the unbound (free) state where it moves by convection and nonFickian diffusion and the bound state where drug attaches reversibly to specific sites inside the plaques and the arterial wall and stays immobilized for a period of time. The concentration of unbound drug in the regions of the arterial wall is represented by $C_{U D, j} j=V, S P, H P$, with initial concentration $C_{U D, j}^{0}=0$, while $B_{\max , j}$ represents the density of free binding sites in the regions of the arterial wall. $C_{B D, j}$ represents the concentration of bound drug with initial concentration $C_{B D, j}^{0}=0$. The drug-binding reaction is schematically represented by

$$
C_{B D, j} \stackrel{\kappa_{b, j}}{\underset{\kappa_{u, j}}{\rightleftarrows}} C_{U D, j},
$$

where $\kappa_{b, j}$ is the association rate between the drug and the binding sites and $\kappa_{u, j}$ is the dissociation rate.

Convective terms As the vessel wall is a porous media, drug transport in the arterial wall is not only governed by diffusion but also depends on the advection induced by plasma filtration in the tissue, activated by physiological transmural pressure gradients.

Let $u_{j}$ and $p_{j}, j=V, S P, H P$, represent the filtration velocity vector of the plasma and the pressure 
in different regions of the arterial wall respectively. The velocity $u_{j}$ is described by Darcy's equation with boundary and interface conditions constrained with the incompressibility condition to ensure mass conservation.

In order to find the pressure drop in the stented arterial wall, we consider that the permeability $k_{j}$ and viscosity $\mu_{j}, j=V, S P, H P$, are constants. So we have the following coupled system in terms of pressure drop:

$$
\begin{cases}-\nabla \cdot\left(\frac{k_{j}}{\mu_{j}} \nabla p_{j}\right)=0 & \text { in } j=V, S P, H P, \\ p_{j}=p_{\text {lumen }}, j=V, S P, H P & \text { on } \Gamma_{\text {wall-lumen }} \cup \Gamma_{\text {soft-lumen }} \cup \Gamma_{\text {hard-lumen }}, \\ p_{V}=p_{j}, j=S P, H P & \text { on } \Gamma_{\text {wall-soft }} \cup \Gamma_{\text {wall-hard }}, \\ \frac{k_{V}}{\mu_{V}} \nabla p_{V} \cdot \eta_{V}=-\frac{k_{j}}{\mu_{j}} \nabla p_{j} \cdot \eta_{j}, j=S P, H P & \text { on } \Gamma_{\text {wall-soft }} \cup \Gamma_{\text {wall-hard }}, \\ \nabla p_{j} \cdot \eta_{j}=0 & \text { on } \Gamma_{\text {coat-wall }} \cup \Gamma_{\text {coat-soft }} \cup \Gamma_{\text {coat-hard }}, \\ p_{V}=p_{\text {adv }} & \text { on } \Gamma_{\text {adv. }} .\end{cases}
$$

For a sake of simplicity, we assume $p_{\text {adv }}=0$ and a nonzero pressure $p_{\text {lumen }}=p_{0}$.

We impose a pressure difference $\delta p=p_{\text {blood }}-p_{\text {adv }}=90 \mathrm{mmHg}$ between the inner and the outer surface of the arterial wall. The values of permeability and viscosity in the arterial wall have been taken from [30].

The viscoelastic effect Arterial walls are known to display complex mechanical responses under physiological conditions. The coronary arterial wall consists of elastin that is responsible for elasticity and smooth muscle cell and collagen in the media, which dictate the viscoelastic behavior of the artery. Experiments like creep test have demonstrated that the vascular tissue is viscoelastic ([11 19 24] $)$. It is accepted that in the presence of small vascular deformations, the linear viscoelastic models will adequately capture the viscoelastic properties of the arterial wall ([19]).

The linear viscoelastic model (Maxwell-Wiechert model, [5]),

$$
\frac{\partial \sigma_{j}}{\partial t}+\frac{1}{\tau_{j}} \sigma_{j}=-\frac{k_{j} \kappa_{r_{j}}}{\tau_{j}}\left(C_{m, j}+\tau_{\sigma_{j}} \frac{\partial C_{m, j}}{\partial t}\right), \text { in } j \times \mathbb{R}^{+}, j=V, S P, H P,
$$

is used in [18, to capture the viscoelastic properties of the healthy wall, soft and hard plaques where $\tau_{j}=\frac{\eta_{j}}{\kappa_{j}}$ and $\tau_{\sigma_{j}}=\eta_{j} \frac{\kappa_{j}+\kappa_{r_{j}}}{\kappa_{j} \kappa_{r_{j}}}, j=V, S P, H P$. The constants $\kappa_{j}$ represent the Young's modulus of the Maxwell arm in each arterial regions while $\eta_{j}$ are their viscosities.

As the hard plaque is stiffer than the soft plaque and the healthy wall, we consider $\kappa_{r_{S P}} \leq \kappa_{r_{V}} \leq \kappa_{r_{H P}}$.

The mathematical model in the arterial wall The non-Fickian nonlinear reaction-diffusionconvection model in the arterial wall reads:

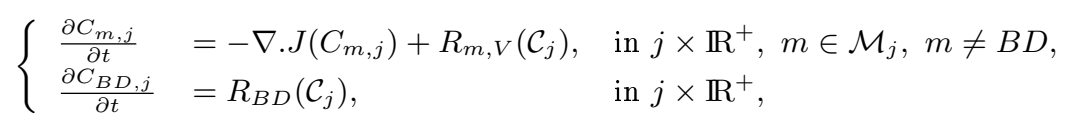

for $j=V, S P, H P$, where

$$
R_{m, j}\left(\mathcal{C}_{j}\right)= \begin{cases}-\mathcal{R}_{1, j}\left(\mathcal{C}_{j}\right), & \mathrm{m}=\mathrm{W} \\ -\mathcal{R}_{1, j}\left(\mathcal{C}_{j}\right), & \mathrm{m}=\mathrm{O} \\ \mathcal{R}_{1, j}\left(\mathcal{C}_{j}\right), & \mathrm{m}=\mathrm{L} \\ \mathcal{R}_{2, j}\left(\mathcal{C}_{j}\right), & \mathrm{m}=\mathrm{BD} \\ -\mathcal{R}_{2, j}\left(\mathcal{C}_{j}\right), & \mathrm{m}=\mathrm{UD}\end{cases}
$$


and

$$
\left\{\begin{array}{l}
\mathcal{R}_{1, j}\left(\mathcal{C}_{j}\right)=k_{O W, j} C_{W, j} C_{O, j}\left(1+\gamma C_{L, j}\right) \\
\mathcal{R}_{2, j}\left(\mathcal{C}_{j}\right)=\varepsilon_{j}^{-1} \kappa_{u, j} C_{U D, j}\left(B_{\max , j}-C_{B D, j}\right)-\kappa_{b, j} C_{B D, j}
\end{array}\right.
$$

for $j=V, S P, H P$. In 18$] \varepsilon_{j}$ stands for the porosity in different regions of the arterial wall $([26])$.

The mass flux in the arterial wall is defined by

$$
J\left(C_{m, j}\right)=-\left(D_{m, j} \nabla C_{m, j}+D_{\sigma_{j}} \nabla \sigma_{j}-u_{j} C_{m, j}\right), m \in \mathcal{M}_{j}, j=V, S P, H P
$$

where $u_{j}$ in 19 is the velocity field computed by Darcy's law (see section 2.3. $D_{\sigma_{j}}, j=V, S P, H P$, represent the non-Fickian diffusion coefficient which acts as a barrier to entrance of the diffusible molecules from the stent $([9],[10],[18])$.

Initial and boundary conditions Equations $(16)-19$ are completed with initial and boundary conditions.

$$
C_{m, j}(0)=C_{m, j}^{0}, m=W, C_{m, j}(0)=0, m=O, L, U D, B D .
$$

As the plasma penetrates from the blood lumen into the arterial wall, we may consider a Dirichlet boundary condition $C_{W, j}=C_{W, \text { out }}, j=V, S P, H P$, on $\Gamma_{\text {lumen-wall }} \cup \Gamma_{\text {lumen-soft }} \cup \Gamma_{\text {lumen-hard }}$, where $C_{W \text {,out }}$ stands for the plasma concentration in the lumen. As oligomers, lactic acid and unbound drug present in the boundary of the arterial wall go directly into the blood and are transported very fast away from the region of interest, the Dirichlet boundary condition $C_{m, j}=0, j=V, S P, H P$, is considered on the lumen boundary $\Gamma_{\text {lumen-wall }} \cup \Gamma_{\text {lumen-soft }} \cup \Gamma_{\text {lumen-hard. }}$. We assume that adventitia is impermeable to all species present in the arterial wall, so the non-flux condition $J\left(C_{m, V}\right) . \eta_{V}=0, m \in \mathcal{M}_{V}, m \neq B D$, holds for $\Gamma_{\text {adv }}$, where $\eta_{V}$ is the exterior unit normal. The calcified plaque is also assumed to be impermeable to all species present in the hard plaque, consequently the non-flux condition $J\left(C_{m, H P}\right) . \eta_{H P}=0, m \neq B D$, on $\Gamma_{\text {calcified }}$ is assumed, where $m \in \mathcal{M}_{H P}$ and $\eta_{H P}$ represents the exterior unit normal.

\subsection{The interface boundary conditions}

As represented in Figure 1 the struts have different deployments depths: completely inserted in the lipid plaque, with a slight penetration in the healthy wall, and on the boundary of the vessel wall in the region with calcium plaque. Interface boundary conditions on $\Gamma_{\text {coat-wall }}, \Gamma_{\text {coat-soft }}, \Gamma_{\text {coat-hard }}, \Gamma_{\text {wall-soft }}$ and $\Gamma_{\text {wall-hard }}$ respectively are defined by

$$
\begin{cases}C_{m, S}=C_{m, V}, & \text { on } \Gamma_{\text {coat-wall }} \\ J\left(C_{m, S}\right) \cdot \eta_{S}=-J\left(C_{m, V}\right) \cdot \eta_{V}, & \text { on } \Gamma_{\text {coat-wall }}\end{cases}
$$

for $m \in \mathcal{M}_{V}, m \neq B D$,

$$
\begin{cases}C_{m, S}=C_{m, S P}, & \text { on } \Gamma_{\text {coat-soft }}, \\ J\left(C_{m, S}\right) \cdot \eta_{S}=-J\left(C_{m, S P}\right) \cdot \eta_{H P}, & \text { on } \Gamma_{\text {coat-soft }}\end{cases}
$$

for $m \in \mathcal{M}_{S}, m \neq P$,

$$
\begin{cases}C_{m, S}=C_{m, H P}, & \text { on } \Gamma_{\text {coat-hard }}, \\ J\left(C_{m, S}\right) \cdot \eta_{S}=-J\left(C_{m, H P}\right) \cdot \eta_{H P}, & \text { on } \Gamma_{\text {coat-hard }},\end{cases}
$$


for $m \in \mathcal{M}_{S}, m \neq P$,

$$
\begin{cases}C_{m, V}=C_{m, S P}, & \text { on } \Gamma_{\mathrm{wall}-\mathrm{soft}} \\ J\left(C_{m, V}\right) \cdot \eta_{V}=-J\left(C_{m, S P}\right) \cdot \eta_{S P}, & \text { on } \Gamma_{\mathrm{wall}-\mathrm{soft}}\end{cases}
$$

for $m \in \mathcal{M}_{S P}$ and

$$
\begin{cases}C_{m, V}=C_{m, H P}, & \text { on } \Gamma_{\text {wall-hard }}, \\ J\left(C_{m, V}\right) \cdot \eta_{V}=-J\left(C_{m, H P}\right) \cdot \eta_{H P}, & \text { on } \Gamma_{\text {wall-hard }},\end{cases}
$$

for $m \in \mathcal{M}_{H P}$.

\section{Numerical Simulations}

The governing equations are discretized in space with the finite element method, using the commercial software package COMSOL Multiphysics 5.1 (COMSOL AB, Burlington, MA, USA).

The coating and arterial wall domains are meshed as illustrated in Figure 2. A finer mesh is used in the coating considering the much smaller scale of the coating domain. Refined meshes in the boundary layers are also defined at the stent-wall interfaces to improve the simulation accuracy.

The time integration is performed with backward differentiation formulae (BDF). Several choices of finite element spaces can be made, but we use here the piecewise quadratic finite element space $P_{2}$ for the concentrations. The average mesh sizes in the stent, in the healthy wall, soft and hard plaques are $2.84 \times 10^{-4} \mathrm{~m}$ (2662 elements), $1.12 \times 10^{-4} \mathrm{~m}$ (3988 elements), $3.25 \times 10^{-3} \mathrm{~m}$ (634 elements) and $3.46 \times$ $10^{-5} \mathrm{~m}$ (4573 elements) respectively. The computational time for the reference simulation performed on an Intel(R) Core(TM) i7-4790 3.60 GHz processor, 16.0 GB RAM and 64-bit operating system is around

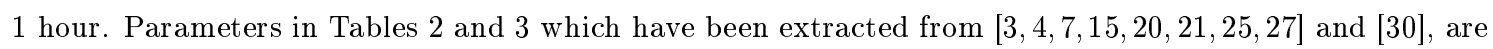

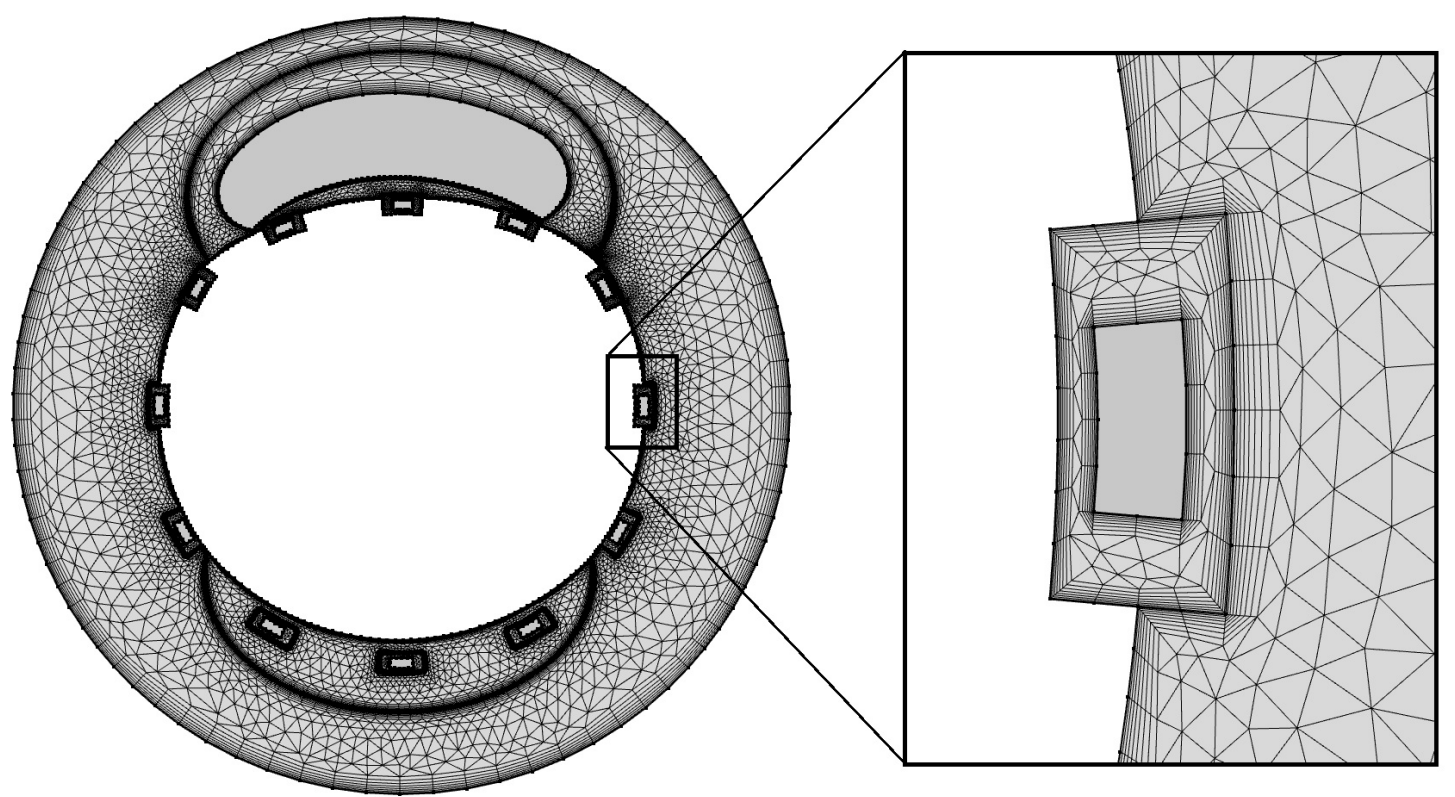

Figure 2: Computational meshes in the domain.

used in all numerical experiments. 


\begin{tabular}{|c|c|c|}
\hline Parameter/Variable & Definition & Value \\
\hline & PLA based stent & \\
\hline$\overline{D_{W, S}^{0}}$ & diffusion coefficient of plasma & $10^{-8} \mathrm{~cm}^{2} / \mathrm{s}$ \\
\hline$D_{M, S}^{0}$ & diffusion coefficient of monomers & $10^{-14} \mathrm{~cm}^{2} / \mathrm{s}$ \\
\hline$D_{D D, S}^{0}$ & diffusion coefficient of dissolved drug & $3.1 \times 10^{-12} \mathrm{~cm}^{2} / \mathrm{s}$ \\
\hline$\kappa_{P W}$ & rate of first reaction & $10^{-6} \mathrm{~cm}^{2} / \mathrm{g} . \mathrm{s}$ \\
\hline$\kappa_{O W}$ & rate of second reaction & $10^{-7} \mathrm{~cm}^{2} / \mathrm{g} . \mathrm{s}$ \\
\hline$k_{D D, S}$ & dissolution rate & $10^{-4} \mathrm{~mol} / \mathrm{cm}^{2} . \mathrm{s}$ \\
\hline$\phi_{S, 0}$ & initial porosity & 0 \\
\hline$C_{\text {Sol }}$ & maximum solubility & $3 \times 10^{-4} \mathrm{~mol} / \mathrm{cm}^{2}$ \\
\hline$\alpha$ & dimensional parameter & $1 \mathrm{~s} / \mathrm{cm}^{2}$ \\
\hline$\beta$ & dimensional parameter & $10 \mathrm{~s} / \mathrm{cm}^{2}$ \\
\hline$\kappa_{S}$ & partitioning coefficient & $10^{-4}$ \\
\hline & Healthy wall & \\
\hline$\overline{D_{W, V}}$ & diffusion coefficient of plasma & $10^{-8} \mathrm{~cm}^{2} / \mathrm{s}$ \\
\hline$D_{M, V}$ & diffusion coefficient of monomers & $10^{-14} \mathrm{~cm}^{2} / \mathrm{s}$ \\
\hline$D_{D, V}$ & diffusion coefficient of drug & $7.7 \times 10^{-8} \mathrm{~cm}^{2} / \mathrm{s}$ \\
\hline$D_{\sigma_{V}}$ & viscoelastic diffusion coefficient & $5 \times 10^{-8} \mathrm{~g} /(\mathrm{cmsPa})$ \\
\hline$\kappa_{O W}$ & rate of reaction & $10^{-7} \mathrm{~cm}^{2} / \mathrm{g} . \mathrm{s}$ \\
\hline$\gamma$ & dimensional parameter & $10 \mathrm{~s} / \mathrm{cm}^{2}$ \\
\hline$\tau_{V}$ & relaxation time & $0.5 \mathrm{~s}$ \\
\hline$\kappa_{r, V}$ & Young's modulus & $1.5 \mathrm{MPa}$ \\
\hline$\kappa_{V}$ & Young's modulus of the arm & $1 M P a$ \\
\hline$k_{V}$ & permeability of the vessel wall & $10^{-15} \mathrm{~cm}^{2}$ \\
\hline$\mu_{V}$ & viscosity of plasma & $5 \times 10^{-2} \mathrm{~g} / \mathrm{cm} . \mathrm{s}$ \\
\hline$p_{\text {lumen }}$ & pressure in lumen & $120 \mathrm{mmHg}$ \\
\hline $\begin{array}{c}p_{\mathrm{adv}} \\
\phi_{V}\end{array}$ & $\begin{array}{l}\text { pressure in adventitia } \\
\text { porosity }\end{array}$ & $\begin{array}{c}30 \mathrm{mmHg} \\
0.61\end{array}$ \\
\hline & Soft plaque & \\
\hline$D_{W, S P}$ & diffusion coefficient of plasma & $10^{-8} \mathrm{~cm}^{2} / \mathrm{s}$ \\
\hline$D_{O, S P}$ & diffusion coefficient of monomers & $10^{-14} \mathrm{~cm}^{2} / \mathrm{s}$ \\
\hline$D_{D, S P}$ & diffusion coefficient of drug & $7.7 \times 10^{-8} \mathrm{~cm}^{2} / \mathrm{s}$ \\
\hline$\kappa_{O W}$ & rate of reaction & $10^{-7} \mathrm{~cm}^{2} / \mathrm{g} . \mathrm{s}$ \\
\hline$\gamma$ & dimensional parameter & $10 \mathrm{~s} / \mathrm{cm}^{2}$ \\
\hline$D_{\sigma_{S P}}$ & viscoelastic diffusion coefficient & $5 \times 10^{-8} \mathrm{~g} /(\mathrm{cmsPa})$ \\
\hline$\tau_{S P}$ & relaxation time & $0.5 \mathrm{~s}$ \\
\hline$\kappa_{r, S P}$ & Young's modulus & $1.2 \mathrm{MPa}$ \\
\hline$\kappa_{S P}$ & Young's modulus of the arm & $1 \mathrm{MPa}$ \\
\hline$k_{S P}$ & permeability of soft plaque & $10^{-15} \mathrm{~cm}^{2}$ \\
\hline $\begin{array}{l}\mu_{S P} \\
\phi_{S P}\end{array}$ & viscosity of plasma & $5 \times 10^{-2} \mathrm{~g} / \mathrm{cm} . \mathrm{s}$ \\
\hline & Hard plaque & \\
\hline$\overline{D_{W, H P}}$ & diffusion coefficient of plasma & $10^{-8} \mathrm{~cm}^{2} / \mathrm{s}$ \\
\hline$D_{O, H P}$ & diffusion coefficient of monomers & $10^{-14} \mathrm{~cm}^{2} / \mathrm{s}$ \\
\hline$D_{D, H P}$ & diffusion coefficient of drug & $7.7 \times 10^{-8} \mathrm{~cm}^{2} / \mathrm{s}$ \\
\hline$\kappa_{O W}$ & rate of reaction & $10^{-7} \mathrm{~cm}^{2} / \mathrm{g} . \mathrm{s}$ \\
\hline$\gamma$ & dimensional parameter & $10 \mathrm{~s} / \mathrm{cm}^{2}$ \\
\hline$D_{\sigma_{H P}}$ & viscoelastic diffusion coefficient & $5 \times 10^{-8} \mathrm{~g} /(\mathrm{cmsPa})$ \\
\hline$\tau_{H P}$ & relaxation time & $0.5 \mathrm{~s}$ \\
\hline$\kappa_{r, H P}$ & Young's modulus & $4.2 \mathrm{MPa}$ \\
\hline$\kappa_{H P}$ & Young's modulus of the arm & $1 M P a$ \\
\hline$k_{H P}$ & permeability of hard plaque & $10^{-15} \mathrm{~cm}^{2}$ \\
\hline $\begin{array}{l}\mu_{H P} \\
\phi_{H P}\end{array}$ & $\begin{array}{c}\text { viscosity of plasma } \\
\text { porosity }\end{array}$ & $\begin{array}{c}5 \times 10^{-2} \mathrm{~g} / \mathrm{cm} . \mathrm{s} \\
0.45\end{array}$ \\
\hline & & \\
\hline
\end{tabular}

Table 2: Values for the parameters and variables in the stent coating, in the arterial wall, soft and hard plaques. 


\begin{tabular}{|c|l|c|c|c|}
\hline Drug & Diffusivity in PLA & Diffusivity in the wall & $B_{\max }$ & $K d=\frac{k_{b}}{k_{u}}$ \\
\hline Paclitaxel & $4.9 \times 10^{-12} \mathrm{~cm}^{2} / \mathrm{s}$ & $2.6 \times 10^{-8} \mathrm{~cm}^{2} / \mathrm{s}$ & $0.127 \mathrm{~mol} / \mathrm{m}^{2}$ & $3.1 \times 10^{-3} \mathrm{~mol} / \mathrm{m}^{2}$ \\
Sirolimus & $3.1 \times 10^{-12} \mathrm{~cm}^{2} / \mathrm{s}$ & $7.7 \times 10^{-8} \mathrm{~cm}^{2} / \mathrm{s}$ & $0.366 \mathrm{~mol} / \mathrm{m}^{2}$ & $2.6 \times 10^{-3} \mathrm{~mol} / \mathrm{m}^{2}$ \\
\hline
\end{tabular}

Table 3: Experimental parameters in the arterial wall for different drugs.

In the numerical simulations Sirolimus is used. In Figure 9 a comparison between Sirolimus an Paclitaxel is exhibited.

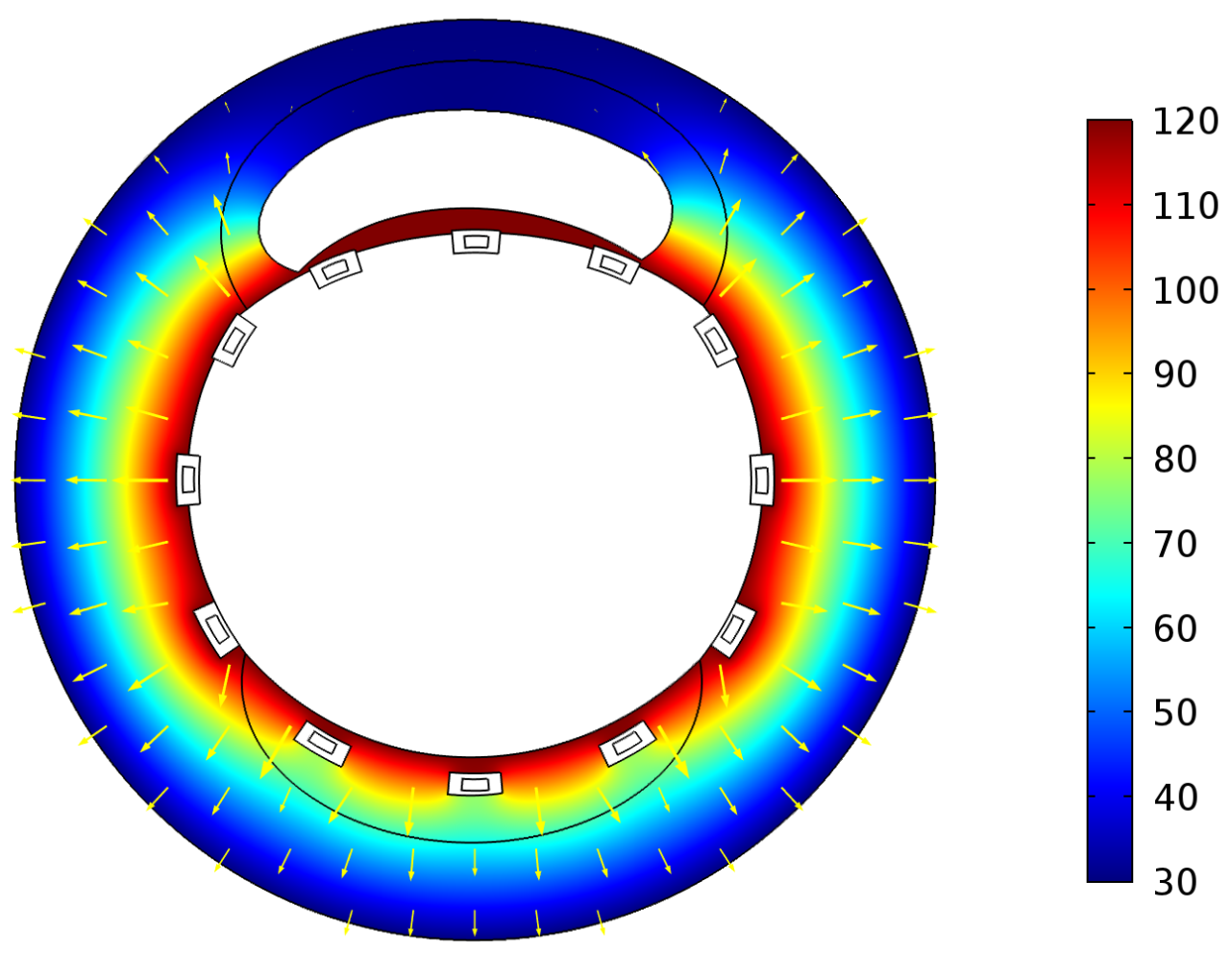

Figure 3: Pressure distribution and velocity field in the stented arterial wall.

\subsection{Numerical simulations in the stent}

In this section, we study the mechanisms of drug release from the polymeric coating into the arterial wall. We begin by presenting in Figure 3 the steady pressure and velocity field in the vessel wall. We note that highest pressures correspond to small regions entrapped between the struts and the arterial wall. Figure 4 shows the degradation of polymeric coating. We observe that its mass vanishes faster in the struts placed in the regions with hard plaques. This is due to the fact that a larger surface of the struts is immersed into the blood flow, consequently more plasma enters inside those struts. 


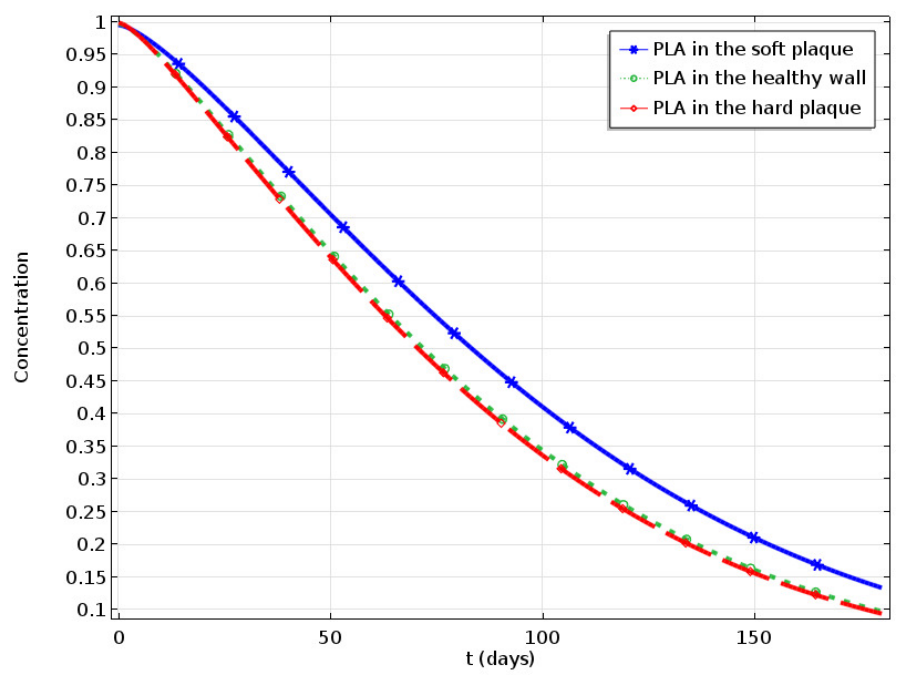

Figure 4: PLA degradation in the stent.

Figure 5 shows the time evolution of the mass of dissolved drug in the polymeric struts embedded in different arterial regions. We observe that the mass of dissolved drug in all struts increases in a first time due to the conversion from solid state to the liquid state and then decreases due to the release of dissolved drug into the different regions of the arterial wall using interface boundary conditions 21 - 23). Struts embedded in the hard plaque have the lowest peaks because they have the largest contact surface with the blood flow. The highest peak of concentration is achieved by struts embedded in the soft plaque.

We conclude from Figure 5 that the position of struts, namely their embedding depth, has an important role in the release of drug.

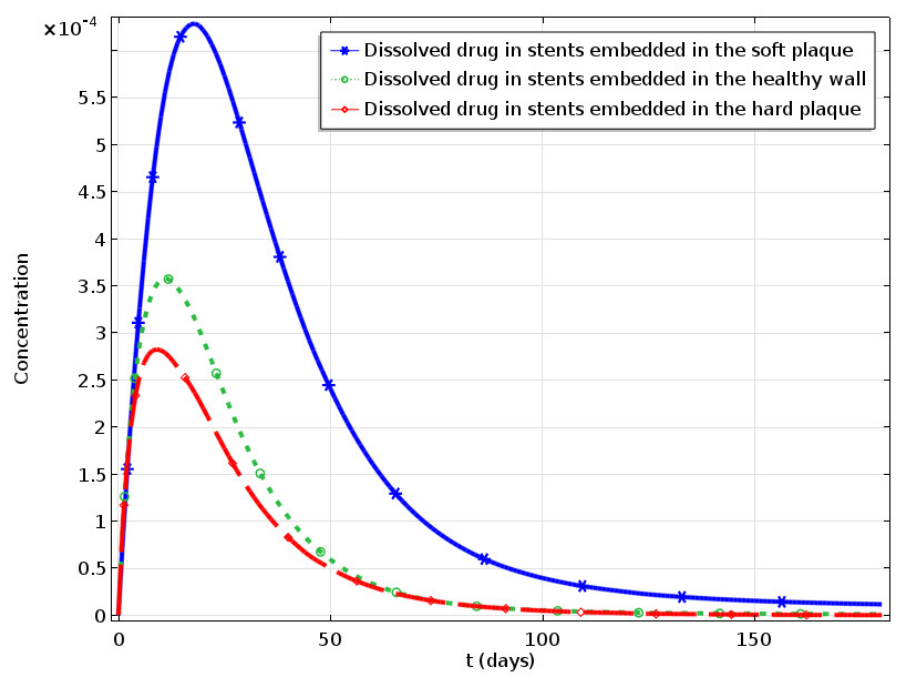

Figure 5: The concentration of dissolved Sirolimus in the stent.

\subsection{Numerical simulations in the arterial wall}

In this section, the mechanism of drug release in the vessel wall is studied. In Figure 6 the time evolution of the mass of unbound Sirolimus in different regions of the vessel wall is illustrated. We observe that the concentration of the unbound Sirolimus in soft plaques is higher than in the other regions. Similar results 
have been obtained for bound drug.

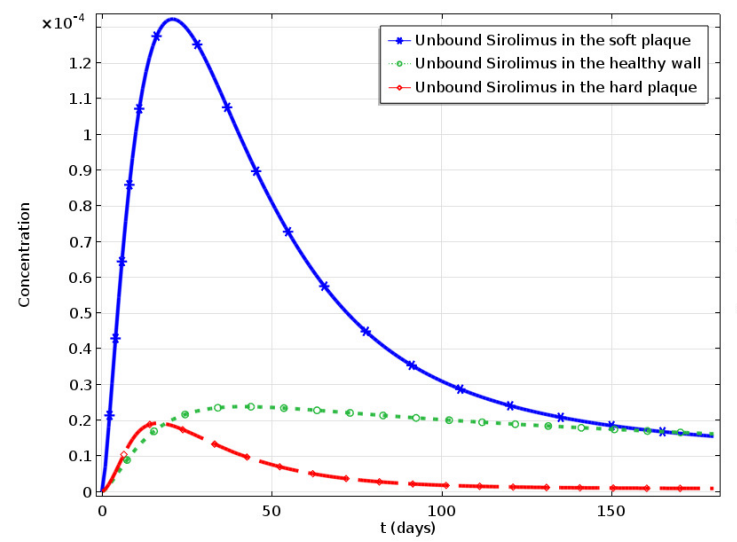

(a) Unbound Sirolimus in the wall.

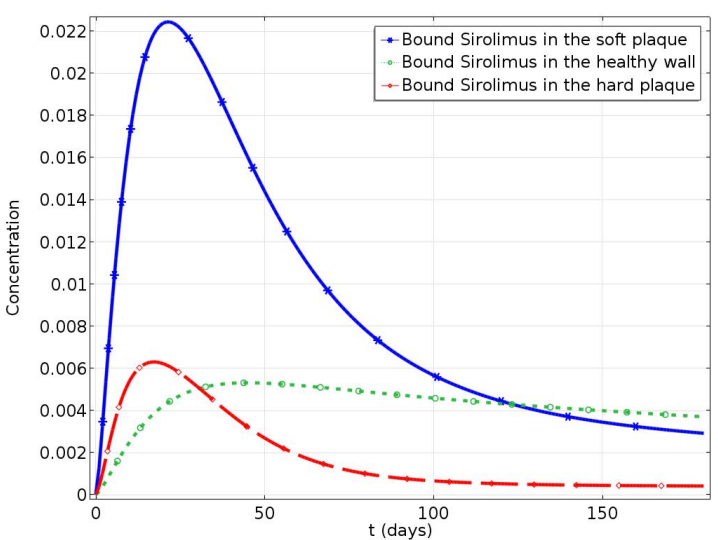

(b) Bound Sirolimus in the wall.

Figure 6: Unbound and bound Sirolimus in the different regions of the vessel wall.

The plots in Figure 6 suggest smaller efficacy in the region of calcium plaques. There are two reasons that can explain this fact. The first is that stents in the stiff region have a larger surface in contact with the blood flow so larger amounts of drug are lost. The second reason is related to the viscoelastic term in equation (19, that represents the hard plaque as a barrier to the penetration of drug. This outcome is in agreement with clinical observation $([6,14])$.

At the best of our knowledge, values for the porosity of the soft and hard plaques are not found in the literature. In Figure 7 we simulate its influence on the concentration of absorbed drug assuming admissible intervals for variation of the porosity. We observe that a larger porosity increases the peak of unbound Sirolimus while it decreases the peak of bound drug in the hard plaque. The reason is that when the porosity increases, the unbound drug can diffuse faster and has less available surface to bind. Analogous results are obtained for the soft plaque.

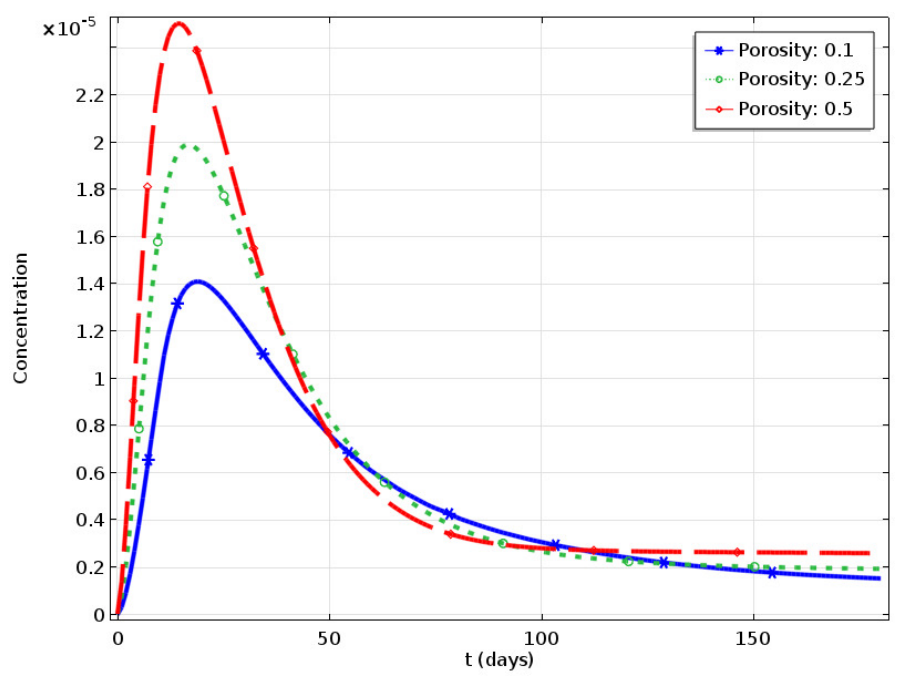

Figure 7: Dependence of the concentration of unbound Sirolimus on the porosity of the hard plaque.

A comparison between Paclitaxel and Sirolimus during three months, after the stent implantation is shown 
in Figure 8 We observe that the concentration of unbound Paclitaxel is larger than the concentration unbound Sirolimus in all the regions of the vessel wall.

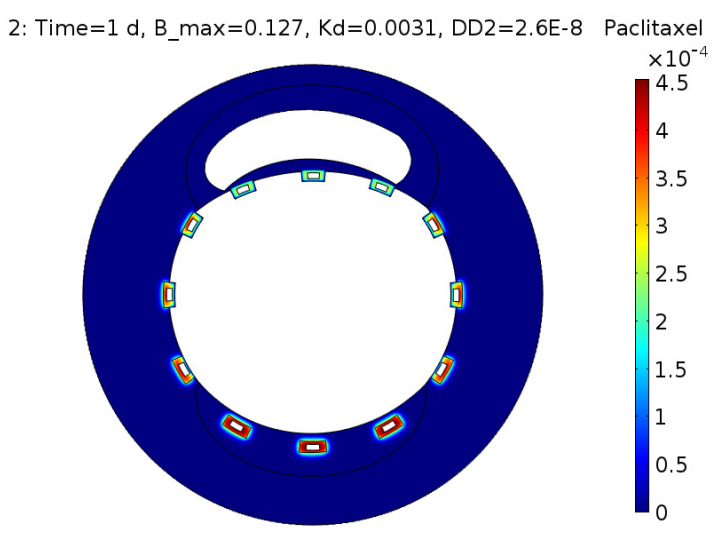

(a) Paclitaxel, 1 day.

31: Time $=30$ d, B_max=0.127, $\mathrm{Kd}=0.0031, \mathrm{DD} 2=2.6 \mathrm{E}-8$

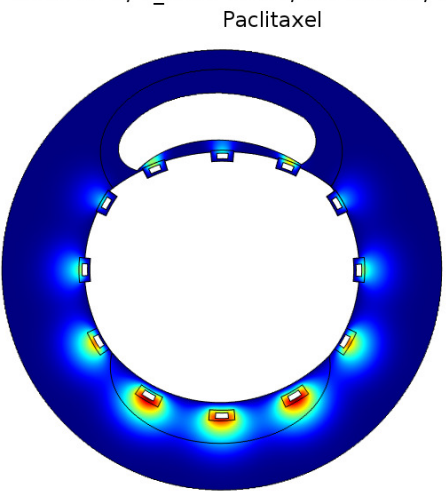

(c) Paclitaxel, 1 month.

91: Time $=90$ d, B_max $=0.127, \mathrm{Kd}=0.0031, \mathrm{DD} 2=2.6 \mathrm{E}-8$

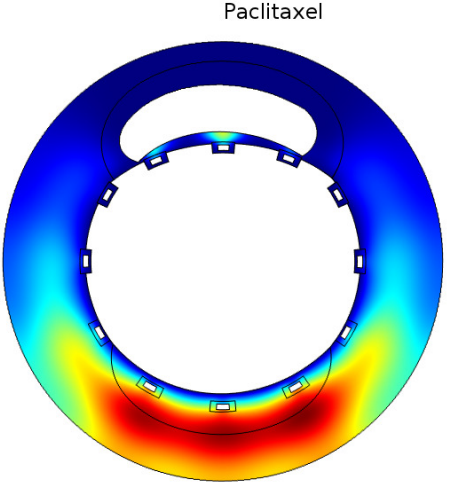

(e) Paclitaxel, 3 months.
183: Time $=1$ d, B_max $=0.366, \mathrm{Kd}=0.0026, \mathrm{DD} 2=7.7 \mathrm{E}-8$

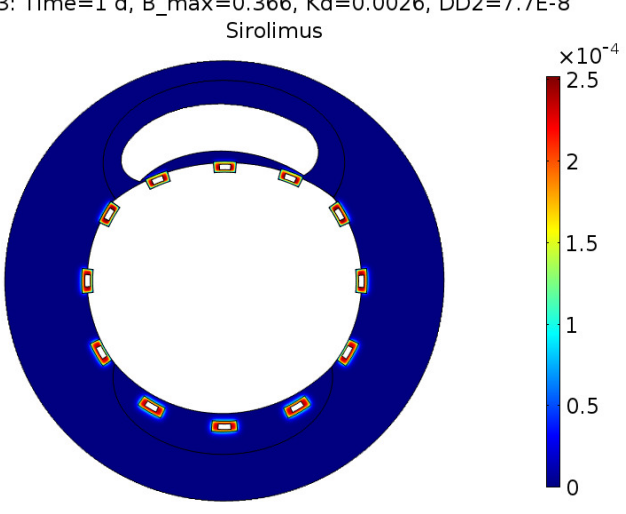

(b) Sirolimus, 1 day.

212: Time $=30$ d, B_max $=0.366, K d=0.0026, D D 2=7.7 E-8$

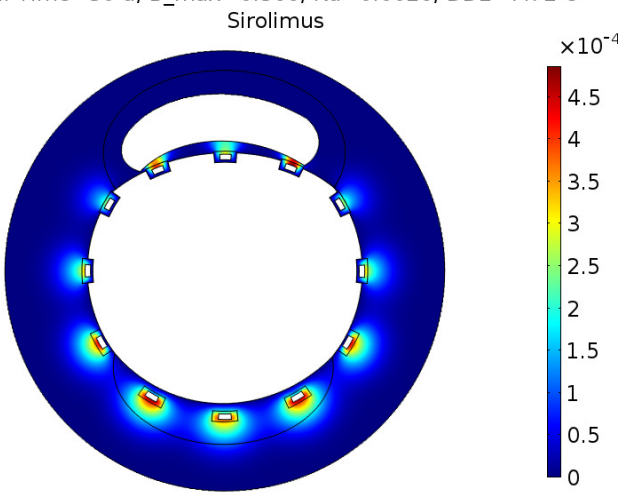

(d) Sirolimus, 1 month. 272: Time $=90$ d, B_max $=0.366, K d=0.0026, D D 2=7.7 E-8$

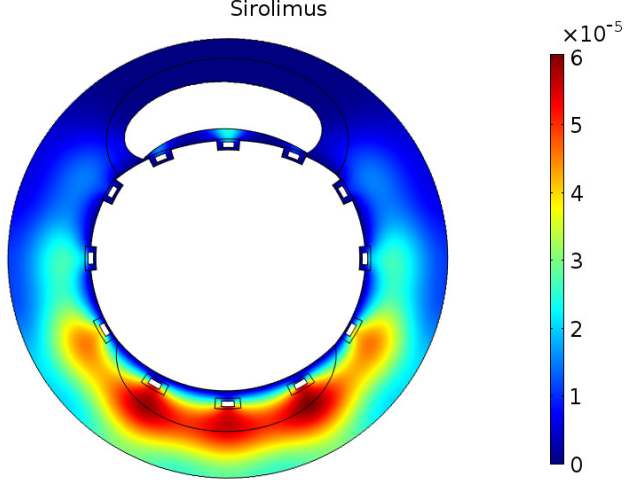

(f) Sirolimus, 3 months.

Figure 8: Unbound drug distribution during 3 months, Paclitaxel vs. Sirolimus.

As in vivo efficacy is defined by the concentration of unbound drug in the target tissue, our results suggest a higher efficacy of Paclitaxel. These last years some papers in the clinical literature have reported a larger incidence of restenosis with Paclitaxel than with Sirolimus. However the results are not conclusive and the controversy is still on going. The data we present can give a contribution to this discussion. 


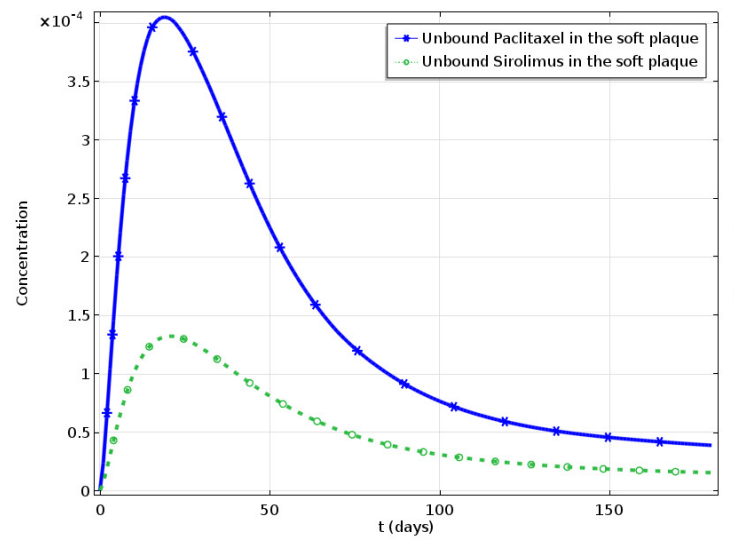

(a) Unbound drug in the soft plaque.

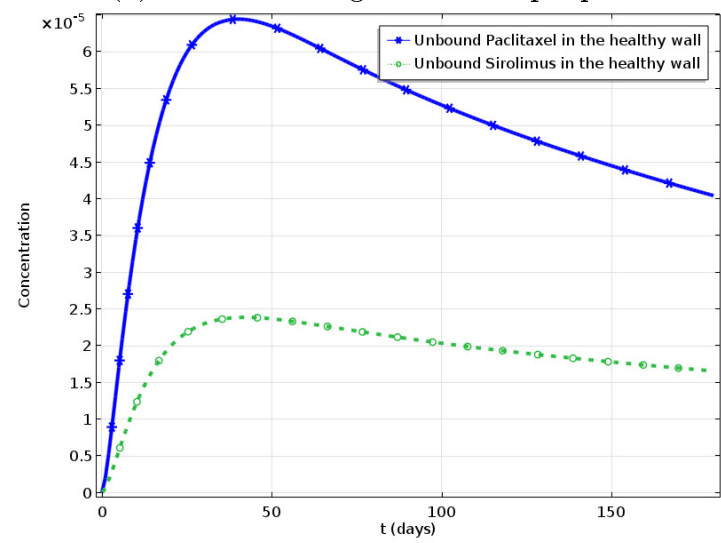

(c) Unbound drug in the healthy wall.

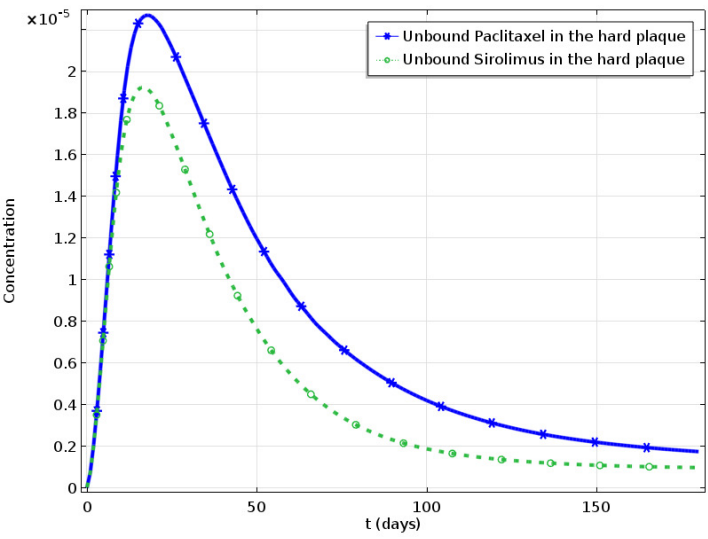

(e) Unbound drug in the hard plaque.

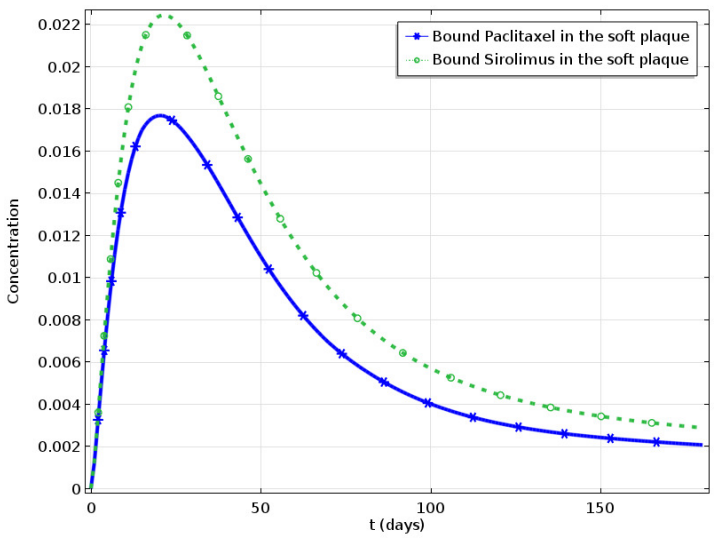

(b) Bound drug in the soft plaque.

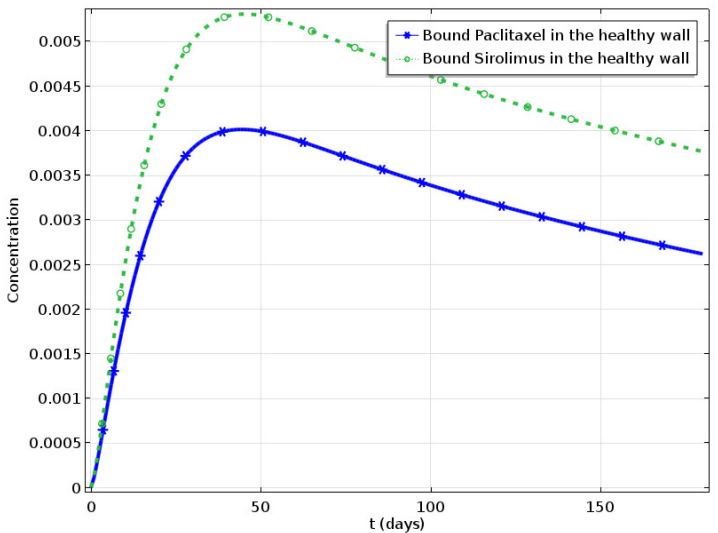

(d) Bound drug in the healthy wall.

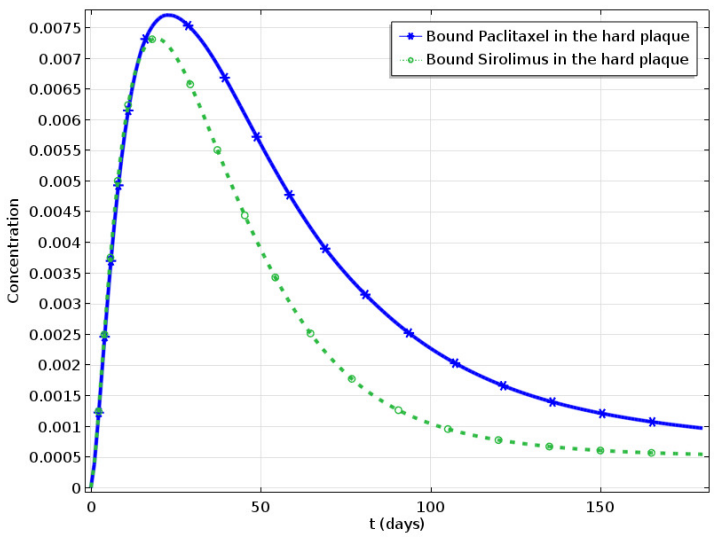

(f) Bound drug in the hard plaque.

Figure 9: Unbound and bound drugs in the arterial wall, Paclitaxel vs Sirolimus.

In fact the effectiveness of a drug depends not only on the total concentration of unbound drug but also on the concentration of bound drug, that defines the residence time. In Figure 9 we represent the bound and unbound concentrations of Paclitaxel and Sirolimus. Although our results suggest a larger residence time of Sirolimus in the soft plaque and the healthy part of the arterial wall, there is an exception in the case of the hard plaque. The cause of this behavior is related to the assumption of the model that there exists a calcified core inside of the hard plaque. To clarify this aspect, we simulate in Figure 10 the distribution 
of the unbound and bound drugs when no calcified core is considered inside of the hard plaque. The immediate finding is that the amount of unbound and bound Sirolimus and Paclitaxel decrease when no calcified core is considered in the hard plaque. A possible reason is that the Neumann condition assumed in the weak formulation of 16$]$ is zero, due to the no-flux condition in the boundary of the calcified core. It represents the fact that unbound drug does not penetrate the calcified core. This term will be removed when no calcified core is considered. Although the concentration of unbound Paclitaxel is higher than the concentration of unbound Sirolimus in the hard plaque, both with and without calcified core, the bound Sirolimus has an higher concentration when no calcified core is assumed in the hard plaque. This means that we can expect a larger residence time of Sirolimus, also in the hard plaques, in case of no calcified core.

A comparison of Figures $9(e)$ and 10 (a) shows that when no calcified core is considered, the highest peak of unbound Sirolimus decreases less than the peak of the unbound Paclitaxel. This means that the unbound Paclitaxel is more sensitive to the existence of the calcified core, leaves faster the hard plaque and consequently Paclitaxel has less time to bind to the tissue. As unbound Sirolimus is less sensitive to these changes, its bound concentration attains an higher peak than the bound Paclitaxel.
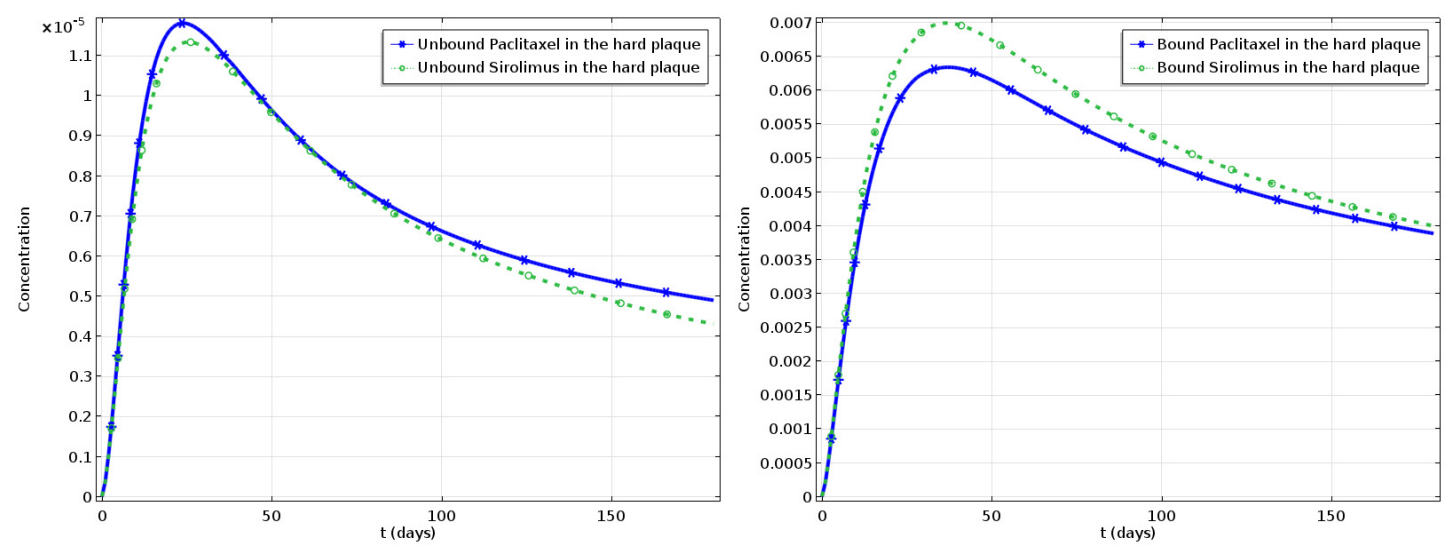

(a) Unbound drug in the hard plaque without cal- (b) Bound drug in the hard plaque without calcified cified core. core.

Figure 10: Unbound and bound drugs in the hard plaque without calcified core, Paclitaxel vs Sirolimus.

Finally we analyze the effect of a topcoat layer in the polymeric coating. When an additional thin layer named topcoat is applied to the polymeric stent, instead of the interface conditions 21$]-(23)$, we consider the following interface conditions

$$
\left\{\begin{array}{l}
J_{m, S} \cdot \eta_{S}=P_{C}\left(C_{m, S}-C_{m, j}\right), \\
J_{m, S} \cdot \eta_{S}=-J_{m, j} \cdot \eta_{j}
\end{array}\right.
$$

for $j=V, S P, H P$, where $P_{C}$ is the permeability of the interface layer between the stent and the wall. The first condition in 26 is the second Kedem-Katchalsky equation (see 20 and the references therein). We remark that the topcoat is used to slow down the release rate of the drug delivery process.

Figure 11 presents the effect of permeability, of the interface layers $\Gamma_{\text {coat-wall }}, \Gamma_{\text {coat-soft }}, \Gamma_{\text {coat-hard }}$, on the drug release when a topcoat is applied to the polymer coating. When a topcoat with smaller permeability is applied to the coated stent, the accumulation of Sirolimus in all wall regions decreases while the accumulation of the dissolved drug in the stent increases. This means that the release of drug from the stent 
into the arterial wall can be controlled by applying topcoats with different permeabilities.
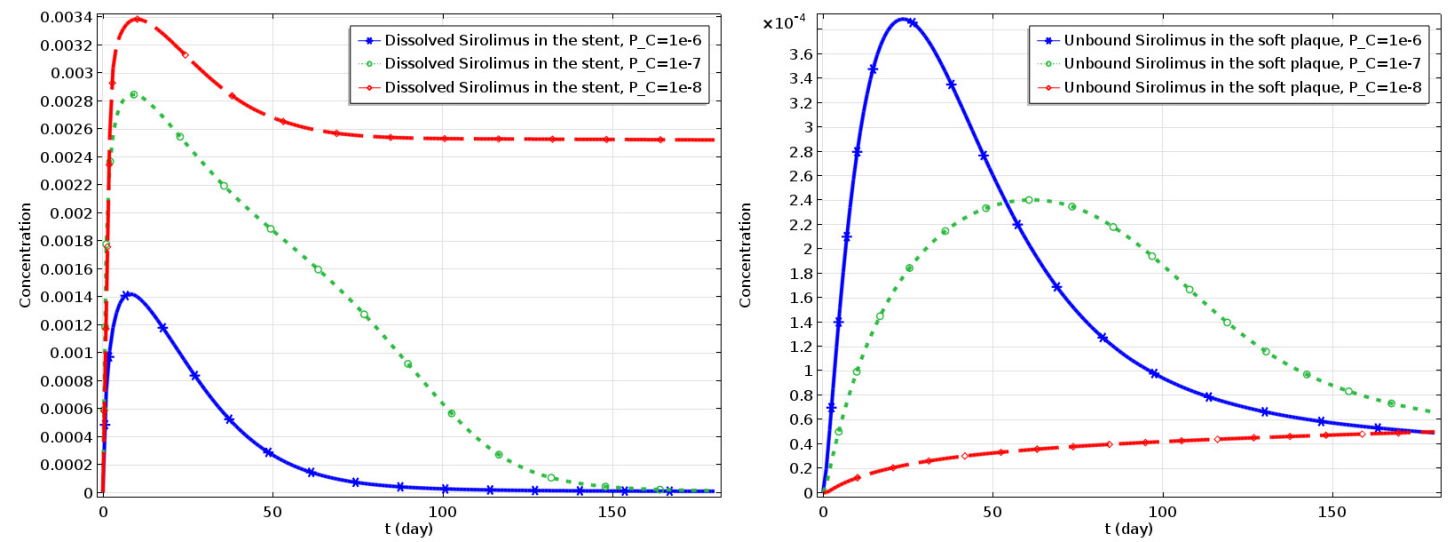

(a) Dissolved Sirolimus in the stent, change in per-

(b) Unbound Sirolimus in the soft plaque, change meability.

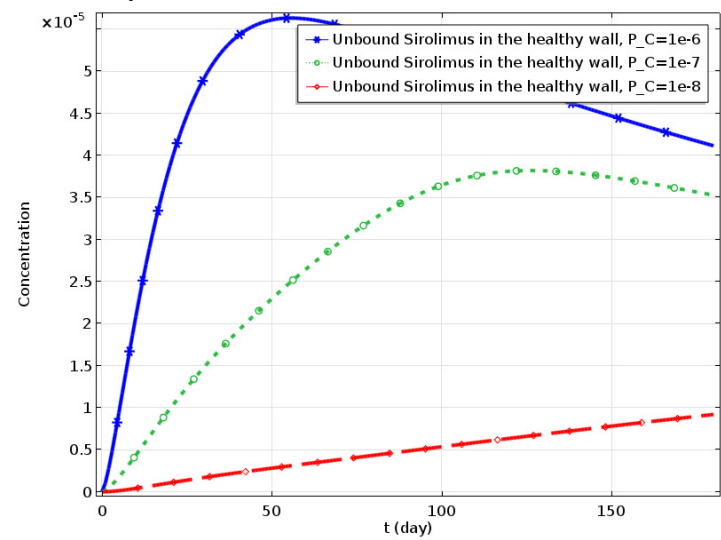
in permeability.

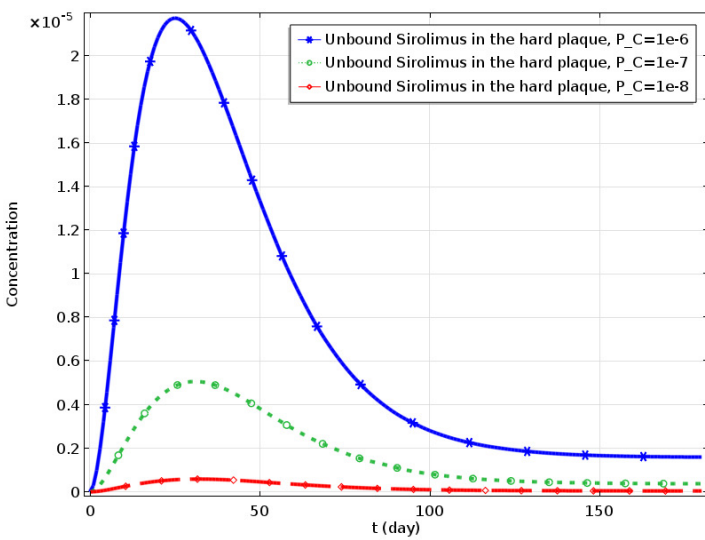

(c) Unbound Sirolimus in the healthy wall, change (d) Unbound Sirolimus in the hard plaque, change in permeability.

in permeability.

Figure 11: The effect of permeability of the interface of stent-wall on the unbound Sirolimus.

\subsection{Geometry obtained from an OCT image: a case study}

In Figures 12 (a) and (b) we present two OCT images, from a patient treated at CHUC-Centro Hospitalar e Universitário de Coimbra- before and after stenting. Lipid and hard plaques are identified in the post stent OCT image. The widening of the lumen, after stent implantation, is clearly observed from the cross and longitudinal sections of the vessel. 


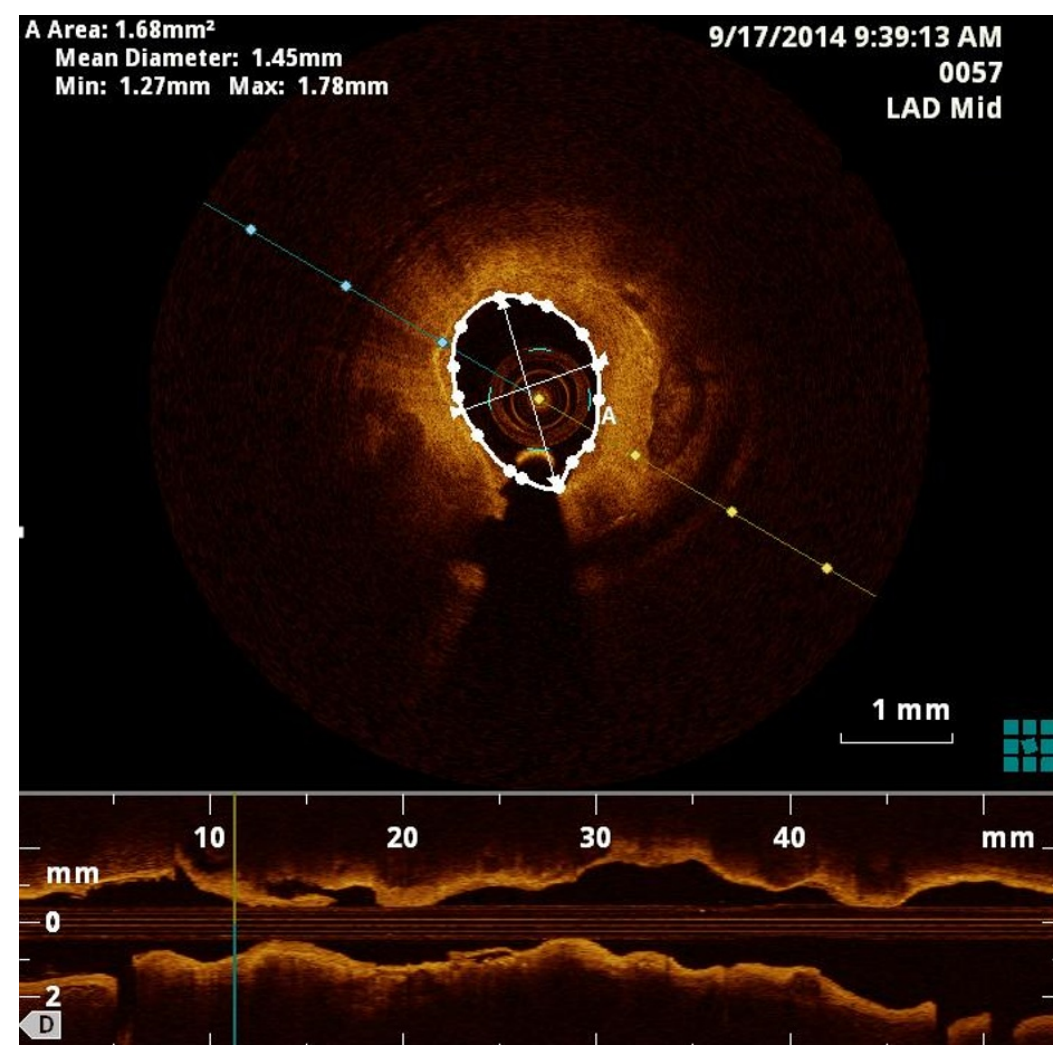

(a) OCT image before stenting,

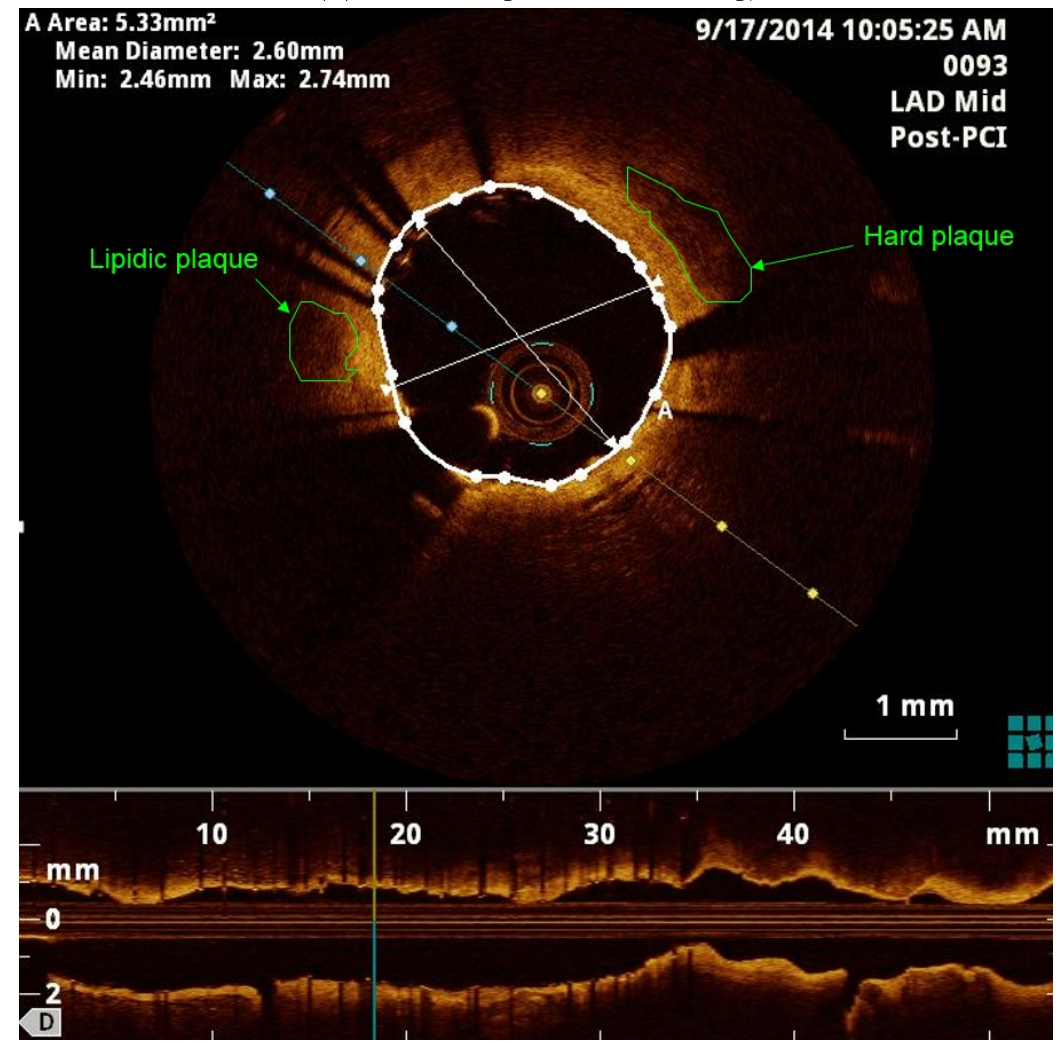

(b) OCT image after stenting,

Figure 12: OCT images before and after stenting. 
Observing Figure 12 we note that there is an incomplete stent apposition, that is an absence of contact between some struts and the vessel wall (see Figure 13. A geometry similar to Figure 12 (b) (the OCT image after stenting), is presented in Figure 13 The lipid and hard plaques as well as the healthy part of the arterial wall are defined as in the OCT image. The stent is a drug eluting stent with a metallic core impermeable to the diffusible species. In this simulation we assume that the diffusible molecules can penetrate into the hard plaque.

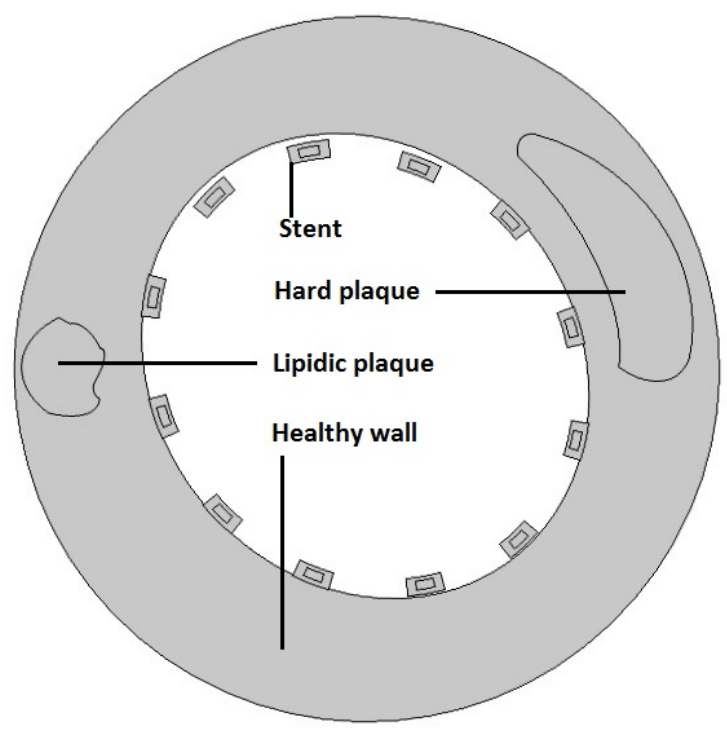

Figure 13: Representation of the geometry in Figure 12 b, where the struts malaposed are identified.

The release of unbound Sirolimus during six months is shown in Figures 14 and 15 . The regions where the struts are malapposed receive less drug when compared to other regions where the stents are embedded or in direct contact. The reason is that the dissolved drug in that case is washed out by the blood flow. In conclusion our results suggest a higher probability of occurrence of restenosis in the regions with hard plaques.

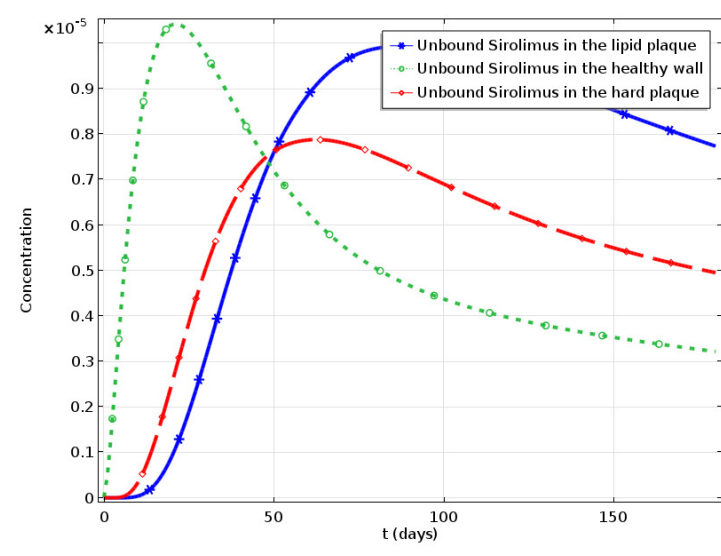

(a) Unbound Sirolimus in the arterial wall

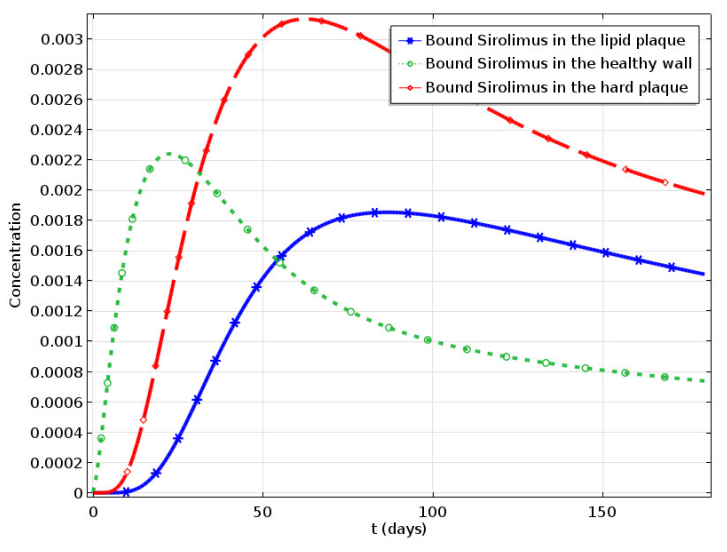

(b) Bound Sirolimus in the arterial wall

Figure 14: Unbound and bound Sirolimus in the arterial wall. 


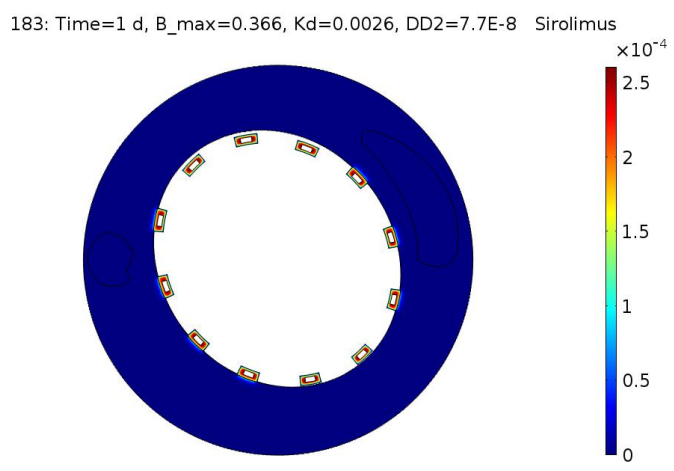

(a) Sirolimus, 1 day,

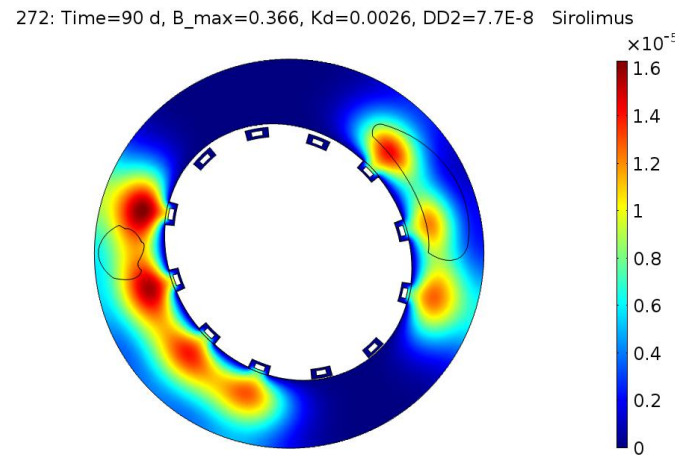

(c) Sirolimus, 3 months,

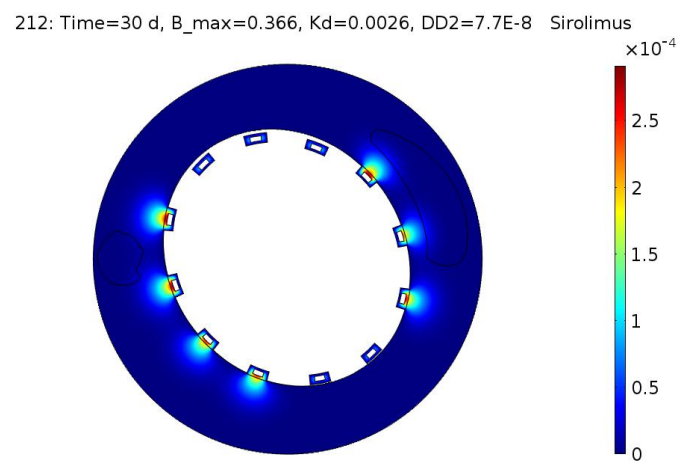

(b) Sirolimus, 1 month,

362: Time $=180 \mathrm{~d}, \mathrm{~B}_{-} \max =0.366, \mathrm{Kd}=0.0026, \mathrm{DD} 2=7.7 \mathrm{E}-8$ Sirolimus

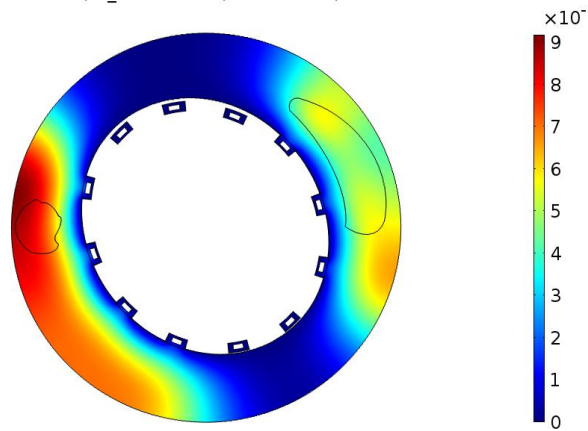

(d) Sirolimus, 6 months,

Figure 15: Distribution of unbound Sirolimus during 6 months.

\section{Conclusions}

In the model presented in this paper we analyze the interplay between the main contributers of drug release from drug eluting stents: the coated stent, the drug, and the arterial wall. Concerning the stent we address the influence of the properties of the bioabsorbable polymeric coating, such as the degradation rate and its time-dependent porosity. The influence of the position of the struts after deployment is also analysed. As far as drugs are concerned we assume that its effectiveness is measured by its efficiency and its residence time. The efficiency is related with the concentration of unbound drug in the target tissue and the residence time depends on the concentration of bound drug. To describe these characteristics of a drug its binding properties are included in the model. Regarding the properties of the atherosclerotic plaque, we refer to the stiffness and porosity of different plaques and its influence on the drug delivery profile as well.

A mathematical model of in vivo drug delivery, although simple when compared with the huge biological complexity of the system, cannot give final conclusive answers, but it can suggest several paths of research. The main objective of the paper is to understand how the pharmacokinetics of a drug is influenced by the stent, the drug itself and the condition of the vessel wall. Our results lead to some preliminary conclusions, regarding not only the separate dependence of these factors but also the dependence on their interaction:

- The degradation of a stent's polymer depends on its position: embedded in the vessel wall, in contact with the vessel wall or malapposed. The position defines the amount of surface in direct contact with blood flow. 
- As less plasma penetrates the struts embedded in the lipid plaque, their degradation is smaller and PLA concentration is larger (Figure 4). Accordingly the concentration of dissolved drug is larger in the struts located in the soft plaque (Figure 5).

- The highest concentration of unbound drug is attained in the soft plaques; the lowest concentration in the region of the hard plaques. Comparing this result with the previous one we conclude that the direct contact with blood flow is a determining factor. Also the fact that hard plaques act like a barrier to the penetration of drug can justify the result (Figure6).

- Comparing the two molecules most used in first generation DES - Paclitaxel and Sirolimus - we conclude that Paclitaxel is more efficient (it presents higher concentrations of unbound drug) but Sirolimus as a larger residence time (it presents higher concentrations of bound drug) in the healthy and lipid plaque but not in the stiff plaque with a calcified core (Figure9).

- The residence time of Sirolimus is also larger than the residence time of Paclitaxel if the plaque has no calcified core (Figure 10 .

- The permeability of the topcoat has a large influence in the drug release (Figure 11).

- The likelihood of occurrence of restenosis is higher in the regions with stiff plaques. This outcome can be explained by the possible malapposition of struts in this region and the more difficult penetration of drug.

Although cardiovascular delivery depends on many others biochemical and physiological phenomena that have not been considered in this paper, we believe that our results can pave the way for a future design of a coadjutant tool in the follow up of stented patients.

\section{Conflict of interest statement}

The authors declare that no conflict of interest occurs.

\section{Acknowledgements}

This work is supported by Alexander von Humboldt Foundation (AvH) under the Georg Forster Research Fellowship (HERMES), hosted at Institute of Structural Mechanics (ISM), Bauhaus-Universität Weimar and partially supported by the Center for Mathematics of the University of Coimbra - UID/MAT/00324/2013, funded by the Portuguese Government through FCT/MEC and co-funded by the European Regional Development Fund through the Partnership Agreement PT2020.

\section{References}

[1] F. Alexis, S. S. Venkatraman, S. K. Rath, and F. Boey, In vitro study of release mechanisms of paclitaxel and rapamycin from drug-incorporated biodegradable stent matrices, Journal of Controlled Release, 98 (2004), pp. 67-74.

[2] E. Azhdari, J. A. Ferreira, P. de Oliveira, and P. M. da Silva, Numerical and analytical study of drug release from a biodegradable viscoelastic platform, Mathematical Methods in the Applied Sciences, (2015). 
[3] F. Bozsak, J.-M. Chomaz, And A. I. Barakat, Modeling the transport of drugs eluted from stents: physical phenomena driving drug distribution in the arterial wall, Biomechanics and modeling in mechanobiology, 13 (2014), pp. 327-47.

[4] F. Bozsak, D. Gonzalez-Rodriguez, Z. Sternberger, P. Belitz, T. Bewley, J.-M. Chomaz, and A. I. Barakat, Optimization of Drug Delivery by Drug-Eluting Stents, PLOS ONE, 10 (2015), p. e0130182.

[5] H. F. Brinson and L. C. Brinson, Polymer Engineering Science and Viscoelasticity: An Introduction, Springer, 2010.

[6] W. M. Camnitz and E. C. Keeley, Heavily calcified coronary arteries: The bane of an interventionalist's existence, Journal of Interventional Cardiology, 23 (2010), pp. 254-255.

[7] M. DErrico, P. Sammarco, and G. Vairo, Analytical modeling of drug dynamics induced by eluting stents in the coronary multi-layered curved domain, Mathematical Biosciences, 267 (2015), pp. 79-96.

[8] J. Ferreira, J. Naghipoor, and P. De Oliveira, Analytical and numerical study of a coupled cardiovascular drug delivery model, Journal of Computational and Applied Mathematics, 275 (2015), pp. $433-446$.

[9] J. A. Ferreira, M. Grassi, E. Gudino, and P. de Oliveira, A $3 D$ Model for Mechanistic Control of Drug Release, SIAM Journal on Applied Mathematics, 74 (2014), pp. 620-633.

[10] J. A. Ferreira, J. Naghipoor, And P. De Oliveira, A coupled non-Fickian model of a cardiovascular drug delivery system, Mathematical Medicine and Biology, 275 (2015).

[11] Y. C. Fung, Biomechanics Mechanical Properties of Living Tissues, Biomechanics / Y. C. Fung, Springer-Verlag, 1993.

[12] S. Garg and P. W. Serruys, Coronary Stents, Journal of the American College of Cardiology, 56 (2010), pp. S1-S42.

[13] P. Gentile, V. Chiono, I. Carmagnola, and P. V. Hatton, An overview of poly(lactic-coglycolic) acid (PLGA)-based biomaterials for bone tissue engineering., International journal of molecular sciences, 15 (2014), pp. 3640-59.

[14] R. E. Kuntz, R. D. Safian, J. P. Carrozza, R. F. Fishman, M. Mansour, and D. S. Baim, The importance of acute luminal diameter in determining restenosis after coronary atherectomy or stenting, Circulation, 86 (1992), pp. 1827-1835.

[15] S. McGinty, A decade of modelling drug release from arterial stents, Mathematical Biosciences, 257 (2014), pp. 80-90.

[16] S. McGinty, M. Wheel, S. MCKee, And C. MCCormick, Does anisotropy promote spatial uniformity of stent-delivered drug distribution in arterial tissue?, International Journal of Heat and Mass Transfer, 90 (2015), pp. 266-279.

[17] S. Minisini, Mathematical and Numerical Modeling of Controlled drug release, phd thesis, Politecnico di Milano, 2009.

[18] J. Naghipoor, J. A. Ferreira, P. D. E. Oliveira, and T. Rabczuk, Tuning polymeric and drug properties in a drug eluting stent: a numerical study, Preprint n. 15-52, Department of Mathematics of Universidade de Coimbra, (2015), pp. 1-32.

[19] A. Nekouzadeh, K. M. Pryse, E. L. Elson, and G. M. Genin, A simplified approach to quasilinear viscoelastic modeling, Journal of Biomechanics, 40 (2007), pp. 3070-3078. 
[20] G. Pontrelli and F. De Monte, A multi-layer porous wall model for coronary drug-eluting stents, International Journal of Heat and Mass Transfer, 53 (2010), pp. 3629-3637.

[21] S. Prabhu and S. Hossainy, Modeling of degradation and drug release from a biodegradable stent coating., Journal of Biomedical Materials Research. Part A, 80 (2007), pp. 732-741.

[22] F. Rossi, T. Casalini, E. Raffa, M. Masi, and G. Perale, Bioresorbable polymer coated drug eluting stent: A model study, Molecular Pharmaceutics, 9 (2012), pp. 1898-1910.

[23] J. S. Soares AND P. Zunino, A mixture model for water uptake, degradation, erosion and drug release from polydisperse polymeric networks., Biomaterials, 31 (2010), pp. 3032-42.

[24] Y. TAO, Nonlinear Viscoelastic Properties and Constitutive Modeling of Blood Vessel, master thesis, National University of Singapore, 2009.

[25] V. Tuor, Mathematical Analysis of Some Models for Drug Delivery, phd thesis, National University of Ireland, Galway, aug 2012.

[26] A. R. Tzafriri, A. D. Levin, and E. R. Edelman, Diffusion-limited binding explains binary dose response for local arterial and tumour drug delivery., Cell proliferation, 42 (2009), pp. 348-63.

[27] G. Vairo, M. Cioffi, R. Cottone, G. Dubini, and F. Migliavacca, Drug release from coronary eluting stents: A multidomain approach, Journal of Biomechanics, 43 (2010), pp. 1580-1589.

[28] R. W. Yeh, S. L. T. Normand, R. E. Wolf, P. G. Jones, K. K. L. Ho, D. J. Cohen, D. E. Cutlip, L. Mauri, A. D. Kugelmass, A. P. Amin, and J. A. Spertus, Predicting the restenosis benefit of drug-eluting versus bare metal stents in percutaneous coronary intervention, Circulation, 124 (2011), pp. 1557-1564.

[29] X. Zhu And R. D. BraAtz, A mechanistic model for drug release in PLGA biodegradable stent coatings coupled with polymer degradation and erosion, Journal of Biomedical Materials Research Part A, 103 (2015), pp. 2269-2279.

[30] P. Zunino, Multidimensional pharmacokinetic models applied to the design of drug-eluting stents, Cardiovascular Engineering, 4 (2004), pp. 181-191. 